\title{
Mobile multicast source support in PMIPv6 networks
}

\author{
Lili Wang ${ }^{1 *}$, Shuai Gao ${ }^{1}$, Hongke Zhang ${ }^{1}$, Thomas C Schmidt ${ }^{2}$ and Jianfeng Guan ${ }^{3}$
}

\begin{abstract}
With the widespread use of multimedia contents via mobile nodes (MNs), IP mobile multicast becomes more important for wireless, mobile, and ubiquitous multimedia applications. Until now, many research efforts have been made to provide IP multicast for the MNs. However, the existing mobile multicast schemes mostly focus on the mobility of receivers based on the host-based mobility solution that requires the MN to participate in the mobility management. Recent work has shown that service connectivity for mobile multicast sources is still a problem and attracts very little concern. With the development of the network-based mobility support protocol, mobile multicast source support schemes in Proxy Mobile IPv6 (PMIPv6) networks are needed urgently. In this paper, we propose a base solution (BS) and also a direct multicast routing scheme (DMRS) for mobile multicast source support in PMIPv6 networks. In the BS, the multicast listener discover (MLD) proxy function is adopted to transmit multicast data through the PMIPv6 tunnel. The DMRS can provide locally optimized traffic flows and avoid inefficient routing present in the BS. We evaluate and compare the performance of the proposed schemes with the Mobile IP bidirectional tunneling (MIP-BT) and Mobile IP remote subscription (MIP-RS) schemes by theoretical analysis and also implement the proposed schemes on the test-bed. The numerical results show that the BS and DMRS outperform the MIP-BT and MIP-RS in terms of signaling cost. Meanwhile, the experimental results verify the feasibility and validity of our proposed schemes. Furthermore, we study the optimal PMIPv6 domain size to reduce the total signaling cost for the proposed schemes.
\end{abstract}

\section{Introduction}

With the rapid development of the Internet, a large number of multimedia services emerge endlessly; and some application services, such as video on demand, television broadcasting, video conferencing, and online distance education, require that multiple subscribers can receive the same data simultaneously. As an important carrier protocol for multimedia, the IP multicast can support data transmission from a source to multiple destinations. However, current IP multicast technology is only applicable to the wired IP network and then is unable to meet the requirements of the wireless and mobile networks. Therefore, the IP mobile multicast with its unique advantages becomes a key technology to solve this problem for the wireless, mobile, and ubiquitous multimedia applications.

*Correspondence: liliwang@bjtu.edu.cn

${ }^{1}$ National Engineering Laboratory for NGI Interconnection Devices, School of Electronic and Information Engineering, Beijing Jiaotong University, No. 3

Shang Yuan Cun, Haidian District, Beijing 100044, China

Full list of author information is available at the end of the article
IP mobile multicast is one of the hot topics in mobile Internet field and has drawn significant attention over a decade [1]. Until now, many approaches have been proposed, but a majority of them are based on the host-based mobility solution [1,2], such as Mobile IPv6 (MIPv6) [3]. MIPv6 requires the mobile node $(\mathrm{MN})$ to modify its client functionality in the IPv6 stack which limits its deployment. Recently, Proxy Mobile IPv6 (PMIPv6) [4], a network-based mobility protocol, is proposed to provide the mobility support for the MNs without the involvement of $\mathrm{MN}$ and then avoids the deployment issue in MIPv6. Therefore, PMIPv6 is believed to be the solution for the future all-IP wireless network [5]. However, PMIPv6 specification does not provide the multicast communication scheme. Consequently, with the emergence of the PMIPv6 protocol, a new development boom for IP mobile multicast has been launched. For example, in order to study the mobile multicast issues based on PMIPv6, the Internet Engineering Task Force (IETF) specifically established a new working group Multicast Mobility (MULTIMOB) in 2009. In addition, current mobile multicast technologies mostly focus on the mobility of the receivers

\section{Springer}

(C) 2013 Wang et al: licensee Springer. This is an Open Access article distributed under the terms of the Creative Commons Attribution License (http://creativecommons.org/licenses/by/2.0), which permits unrestricted use, distribution, and reproduction in any medium, provided the original work is properly cited. 
based on MIPv6, and there are only a few relevant schemes for multicast source mobility.

Notably, compared with the mobility of multicast receiver, the multicast source mobility is also a very important issue for the deployment of the multicast service but more complicated. On one hand, current Internet has the trends from server-centric to user-centric, highly interactive group applications, like user-generated streaming and conferencing [6]. To realize the above applications, one of the core supporting schemes is the multicast source mobility. On the other hand, the mobile source problem has its unique characteristics and is different from the receiver mobility, that is, the mobility of a multicast receiver only has a local and single impact on the receiver, while the source mobility directly results in the failure of the entire multicast tree [1]. Therefore, it is urgent to expand the research to promote the deployment and application of the mobile multicast source technology.

In this paper, a base solution (BS) and a direct multicast routing scheme (DMRS) are proposed to support the mobile multicast source in PMIPv6 networks. In the BS, the multicast listener discover (MLD) proxy [7] functions are deployed at the mobile access gateways (MAGs) to enable the multicast support for mobile sources within a PMIPv6 domain. This base deployment is the simplest way to PMIPv6 multicast extensions, and standard software functions need to be activated on PMIPv6 entities, only at the price of possibly non-optimal multicast routing. Besides, the BS is divided into the source registration (SR) case and the shortest path tree (SPT) case based on RFC4601 [8]. The DMRS is proposed to make a local content distribution service with locally optimized traffic flows for the visited networks and avoid the inefficient routing issue present in the BS. The numerical analysis results show that the DMRS achieves better performance in terms of signaling cost than the BS in the SR case for its optimized routing, whereas with the increment of the path length for the DMRS, the BS in the SPT case outperforms the DMRS in terms of signaling cost. At the same time, all the schemes in the SPT case have lower signaling cost than those in the SR case, and the total signaling costs in the BS and DMRS are lower than the Mobile IP bidirectional tunneling approach (MIP-BT) and Mobile IP remote subscription approach (MIP-RS) [3]. Meanwhile, a small PMIPv6 domain has the advantage of reducing the total signaling cost for the proposed schemes in the case of the static MN, while a large PMIPv6 domain has the benefit for reducing the total signaling cost in the case of the dynamic MN. In addition, we design and implement our proposed schemes on the test-bed and also conduct the evaluation experiments, which shows that the BS in the SPT case has shorter multicast disruption time and lower packet loss on handover than the DMRS. In this way, as an important carrier protocol for multimedia, the
IP mobile multicast can provide better performance for wireless, mobile, and ubiquitous multimedia applications. Now, our proposed approaches in this paper have been accepted as the IETF MULTIMOB working group draft [6].

The remainder of this paper is organized as follows: Section 2 briefly reviews the related work on the multicast mobility schemes based on the host-based and network-based mobility management protocols. Section 3 describes the BS with the deployment of the MLD proxy functions and the DMRS for mobile multicast source support in PMIPv6 networks in detail. Section 4 presents the performance evaluation in terms of signaling costs for the BS, DMRS, MIP-BT, and MIP-RS in multicast video multimedia services. Section 5 presents the implementation overview and the experimental results on the performance of multicast disruption time and packet loss on handover for our proposed schemes. Section 6 concludes the paper.

\section{Related work}

The mobile multicast has been developed for more than 10 years [1], and many approaches have been raised until now. Since IETF has proposed two types of mobility support protocols which are host-based and network-based mobility management architectures, we also divide the current mobile multicast technologies into two categories, that is, the host-based and network-based schemes. In addition, the proposal and research of the mobile multicast technology are committed to solve the problem of the mobility for all the nodes which participate in the multicast service, including the multicast receivers and the multicast sources [2]. Therefore, we conclude the current mobile multicast schemes shown in Table 1. From Table 1, it can be seen that current mobile multicast technologies mostly concern on the mobility of the receivers based on MIPv6, and there are few supporting schemes for mobile multicast source based on PMIPv6.

MIP-BT and MIP-RS [3,9] are proposed as two essential host-based multicast mobility approaches. However, there are still larger defects in both of the mechanisms, such as the triangle routing and tunnel convergence problem [29] in the MIP-BT and higher handover latency and 'outof-synch' problem [10] in the MIP-RS. Therefore, huge time and energy are invested to study the mobile multicast issues and then a variety of solutions, such as mobile multicast (MoM) [11] and range-based mobile multicast

Table 1 Current mobile multicast schemes

\begin{tabular}{lll}
\hline Scheme & MRM $^{\mathbf{a}}$ & MSM $^{\mathbf{b}}$ \\
\hline Host-based & {$[3,9-16]$} & {$[3,9,17-24]$} \\
Network-based & {$[25-28]$} & {$[26]$, this paper [6] } \\
\hline
\end{tabular}

${ }^{a} \mathrm{MRM}$, multicast receiver mobility; ${ }^{\mathrm{b}} \mathrm{MSM}$, multicast source mobility. 
(RBMoM) [12], have been proposed to improve the overall performance, which focus on the defects existed in the two basic algorithms. Besides, there are also some extension methods, including tree morphing approach [17-19] and state update mechanism [20].

MoM [11] mainly addresses the issue of the tunnel convergence problem for the multicast receiver mobility by selecting only one of the home agents (HAs) among a given set of HAs as the DMSP (designated multicast service provider). Other HAs stop sending packets through their outgoing tunnel to the foreign agent (FA). RBMoM [12] trades off the shortest delivery path and the frequency of the multicast tree reconfiguration for the multicast receiver mobility, and, actually, MIP-BT and MIP-RS are the extremes of RBMoM. Therefore, the issue of the mobile multicast source support is not considered and they are all host-based schemes.

In [21], the authors consider the case that MN is working as a source as well as a receiver for the group. For the source, a reverse tunnel from the MN's current point of attachment to its HA is used to forward multicast packets. For the receiver, the MIP-RS is used to receive multicast traffic. That is, a combination of the MIP-BT and MIP-RS is adopted to solve the problem for the $\mathrm{MN}$ as a source and at the same time as a receiver. This scheme is achieved by sending a multicast join message and a notify message from the source to its HA or FA, which brings a high requirement for the hosts and increases the bandwidth resources and signaling overheads.

In [17-19], the authors propose a tree morphing protocol for mobile multicast sources, which reuses and modifies the existing source-based distribution trees to continuously serve for data transmission of mobile sources. By maintaining (CoA, G, HoA) address triples in router states, all nodes are able to simultaneously identify (HoA, G)-based group membership and (CoA, G)-based tree topology. This scheme requires all the routers to be extended, which increases the complexity and introduces an expensive signaling and state refresh costs. Besides, this is a host-based mobile multicast source scheme, which inherits all the disadvantages of MIPv6.

In [20], a state update mechanism for reusing major parts of prior constructed multicast trees is introduced. However, in this scheme, the reestablishment of the multicast tree is not initiated by receiver but by multicast source, which changes a lot for multicast. It is a big issue for all the multicast routers and receivers how to learn the relationship between the home address (HoA) and different care-of addresses (CoAs).

In [22], an extension to the MLD multicast protocol and multicast delivery agent (MDA) node are introduced. The MDA entity is similar to the MAG in PMIPv6, and the tunnel is established between the HA and MDA. Then the data sent by the multicast source are not directly transmitted to the HA, but rather sent to the HA through the MDA. In fact, this scheme is the compromise for the MIP-BT and MIP-RS, for the route is more optimized than that of the MIP-BT and the delay for the reconstruction trees is smaller than that of the MIPRS. However, the signaling interaction is needed between the MDA and multicast source, which brings the extra network load. In addition, the checking of the direct connection issue between the MDA and multicast source is still up in the air, which needs further study and research.

All the above mechanisms are based on the hostbased mobility solution, which has the limitation for the deployment of mobility services for its requirements of MNs. Consequently, with the release of the network-based mobility protocol in the IETF, a new development boom of IP mobile multicast has been launched. In order to provide guidance for supporting multicast in PMIPv6 networks since PMIPv6 specification does not provide the multicast communication scheme, IETF established the MULTIMOB working group in 2009, and the base deployment for mobile multicast receiver support in PMIPv6 domains has been released as RFC6224 [25]. However, there are only a few relevant schemes to support the mobile multicast source based on PMIPv6, and D. von Hugo et al. proposed that it is needed to address the mobile multicast source support in PMIPv6 networks in the future work [30]. Therefore, we dedicate ourselves to study the mobile multicast source support issue, propose some solutions, and submit the corresponding drafts to the IETF [6,3133]. Now, our proposed approaches in this paper have been accepted as the IETF MULTIMOB working group draft [6].

In [26], two PMIPv6 multicast methods are proposed, called the MAG-based method and local mobility anchor (LMA)-based method. However, these two methods are not very good in terms of the feasibility through the validation of our experiments. For the LMA-based method, since RFC3810 [34] specifies the source address of the report message sent by the receivers is the link address, it is infeasible for the receiver to send the report message with the source address which is a globally routable address. In addition, the multicast data sent by the multicast source $\mathrm{MN}$ cannot go through the bidirectional tunnel established by PMIPv6 because current PMIPv6 specification mainly concerns on the mobility support for unicast routing and does not describe the multicast data forwarding scheme. For the MAG-based multicast source mobility approach, if there is no improvement for current scheme, the join message from receivers cannot be sent to the MAG directly. The reason is that the LMA is the topological anchor for the $\mathrm{MN}$, thereby the join message will be sent to the LMA rather than the MAG. 


\section{Proposed schemes}

In this section, two schemes supporting the mobile multicast source in PMIPv6 networks are proposed, which are the BS and the DMRS.

\subsection{Base solution for mobile multicast source support in PMIPv6 networks (BS)}

In the BS for multicast source mobility, just as same as the BS for receiver mobility in RFC6224 [25], the MLD proxy functions are deployed at the MAGs to distribute multicast data in PMIPv6 networks. The MLD proxy instance serving a mobile multicast source $(\mathrm{MN})$ configures its upstream interface at the tunnel towards the MN's corresponding LMA. This base deployment is the simplest way to PMIPv6 multicast extensions in the sense that it follows the common PMIPv6 traffic model without the requirements of new protocol operations and additional infrastructure entities.

Figure 1 shows the reference scenario of the BS for mobile multicast source support in PMIPv6 networks, where the LMAs serve as multicast anchor points and the MAGs not only play the role of first-hop access routers serving multiple MNs on the downstream, but also run an MLD proxy instance for every LMA upstream tunnel. In Figure 1, the MN1 and MN3 are mobile multicast sources, whereas the MN2 and all the correspondent nodes (CNs) are multicast receivers. In this scenario, mobile sources always remain agnostic of multicast mobility operations.

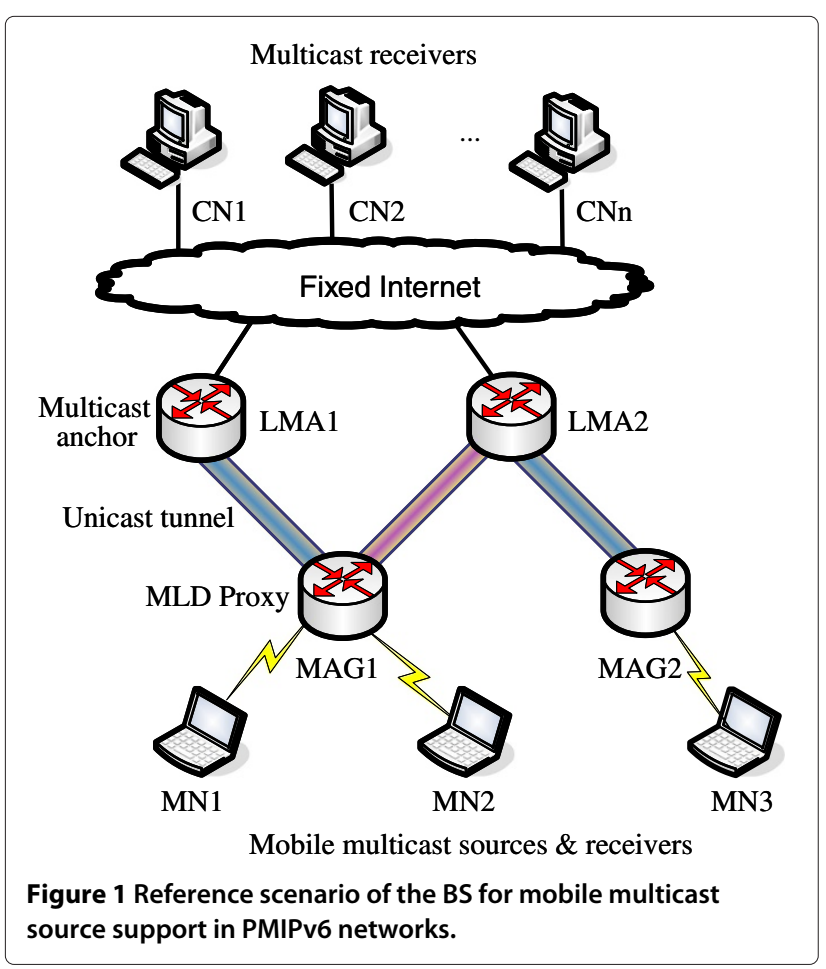

As specified in RFC4605 [7], the multicast data originated from the MN1 will firstly arrive at the MAG1 and then arrive at the LMA1 and directly at the MN2 attached to the same MAG with the MN1 via the MLD proxy function at the MAG1. Serving as the ProtocolIndependent Multicast (PIM) designated router (DR), the LMA1 will firstly encapsulate the multicast packets and forward the data to the virtual interface with encapsulation target rendezvous point (RP) $(G)$, which is the SR case. After receiving the SR packets, the RP will decapsulate and natively forward the packets down the RP-based distribution tree towards the receivers and also initiate a source-specific join for creating a SPT to the mobile source $\mathrm{MN1}(\mathrm{S})$ and issue a source register stop at the native arrival of data from $\mathrm{S}$. Since the LMA1 is the topological anchor point of the mobile source MN1 in the PMIPv6 network, the $(S, G)$ tree will proceed from the RP via the LMA1 and then the LMA1-MAG1 tunnel to the mobile source, which is the SPT case. In response to an exceeded threshold of packet transmission, the DRs of receivers will initiate a source-specific join for creating a SPT to the mobile source $S$, thereby the $(S, G)$ tree will proceed from the receiving DR via the LMA1 and then the LMA1-MAG1 tunnel to the mobile source, which is the source-specific multicast (SSM) case.

When the multicast source MN1 moves from the MAG1 to the MAG2, it can continue to send multicast packets as soon as network connectivity is reconfigured. At this time, the MAG2 firstly determines whether the MN1 is admissible to multicast services and then performs the binding registration to the LMA1, including the IPv6 unicast address configuration. Besides, the MAG2 adds the new downstream link to the MLD proxy instance with upstream link to the LMA1. Therefore, when the MN1 accesses to the MAG2, multicast packets arriving at the MAG2 will be forwarded again to the LMA1. Because the LMA1 is always the DR for the mobile multicast source $\mathrm{MN1}$, the multicast data will eventually be forwarded to the receivers by the LMA1 according to the forwarding states maintained by multicast routing. The detailed handover process call flow is shown in Figure 2, in which 'MLD Membership Report' is abbreviated by 'Join.' As illustrated in Figure 2, when the MN1 attaches to the MAG1, the multicast packets sent by it could be delivered to the CNs through the LMA1 and directly sent to the MN2 attached to the same MAG with the MN1 via the MLD proxy function at the MAG1. When the MN1 hands over to the MAG2, as soon as the binding update to the LMA1 and MLD proxy downstream and upstream interface configuration at the MAG2 has been processed and also the IP address at the MN1 has been configured, the multicast packets could be successfully transmitted by the MAG2 to 




Figure 2 Call flow for group communication in multicast-deployed PMIPv6 networks.

the LMA1 and then to the corresponding receivers $\mathrm{CNs}$ and MN2. In this way, the multicast source mobility is transparently enabled in multicast-deployed PMIPv6 networks.

Besides, the LMA can serve as an additional MLD proxy. If the LMA is acting as another MLD proxy, it will forward the multicast data to its upstream interface and to downstream interfaces with matching subscriptions, accordingly.

These multicast deployment considerations likewise apply for the MNs that operate with their IPv4 stack enabled in PMIPv6 networks. RFC5844 [35] provides the IPv4 home address mobility support in PMIPv6 networks, and an Internet Group Management Protocol (IGMP) proxy function at the MAG can support IPv4 multicast in an analogous way.

However, there exists routing inefficiency problem in this solution. As shown in Figure 1, if the mobile receiver MN2 attaches to the same MAG1 as the mobile source MN1 but associates with a different LMA, the multicast traffic has to flow up to the LMA1, cross over to the LMA2, and then be tunneled downwards to the MAG1, causing redundant flows in the access network and at the MAG1.

\subsubsection{Operations of the $M N$}

For the MN, no specific mobility or other multicast related functionalities are required. Therefore, as a multicast source, an MN willing to send multicast data will proceed as if attached to the fixed Internet.

\subsubsection{Operations of the MAG}

For a MAG, the MLD proxy instances are required to deploy, one for each tunnel to an LMA serving as its unique upstream link. Upon the arrival of an MN, the MAG decides on the mapping of downstream links to a proxy instance and the upstream link to the LMA according to the regular binding update list, for example, that is maintained by PMIPv6 standard operations. According to the specification in RFC4605 [7], when multicast data are received from the MN, the MAG must identify the corresponding proxy instance from the incoming interface and forwards these data to the corresponding upstream link.

\subsubsection{Operations of the LMA}

The LMA, acting as the persistent $\mathrm{HA}$ for the $\mathrm{MN}$ and also as the default multicast upstream for the corresponding MAG, should manage and maintain a multicast forwarding information base for all group traffic arriving from its mobile sources. At the same time, it should participate in multicast routing functions that enable traffic redistribution to all adjacent routers within the PMIPv6 domain and thereby ensure a continuous session when the source is in motion. 


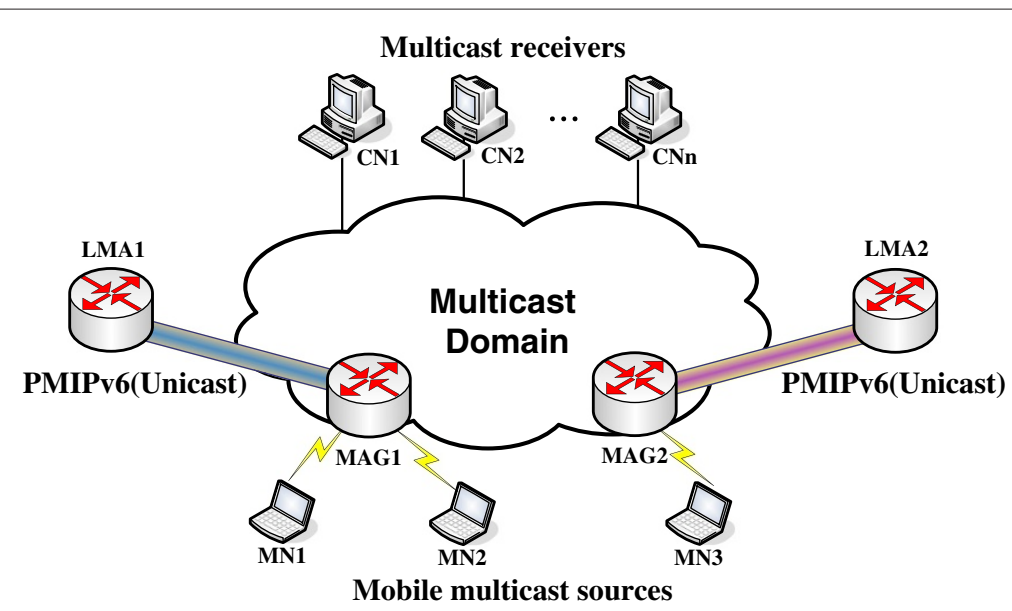

Figure 3 Deployment scenario of PIM-SM at the MAGs for the DMRS.

Besides, according to the specification in RFC4601 [8], as the DRs of the multicast sources, the LMAs operating the Protocol-Independent Multicast-Sparse Mode (PIM$\mathrm{SM}$ ) routing protocol require the sources to be directly connected with itself for sending PIM registers to the RP. However, this does not hold in a PMIPv6 domain, as the MAGs are routers intermediate to the MNs and the LMAs. In this sense, the MNs are multicast sources external to the PIM-SM domain. To mitigate this incompatibility common to all subsidiary MLD proxy domains, we set the LMAs as PIM border routers and activate the border-bit. Notably, running bidirectional ProtocolIndependent Multicast (BIDIR-PIM) [36] on the LMAs can also address this issue and does not require a special configuration.

\subsection{Direct multicast routing scheme for mobile multicast source support in PMIPv6 networks (DMRS)}

As described in Section 3.1, all the multicast data stream must firstly arrive at the LMA and then be forwarded

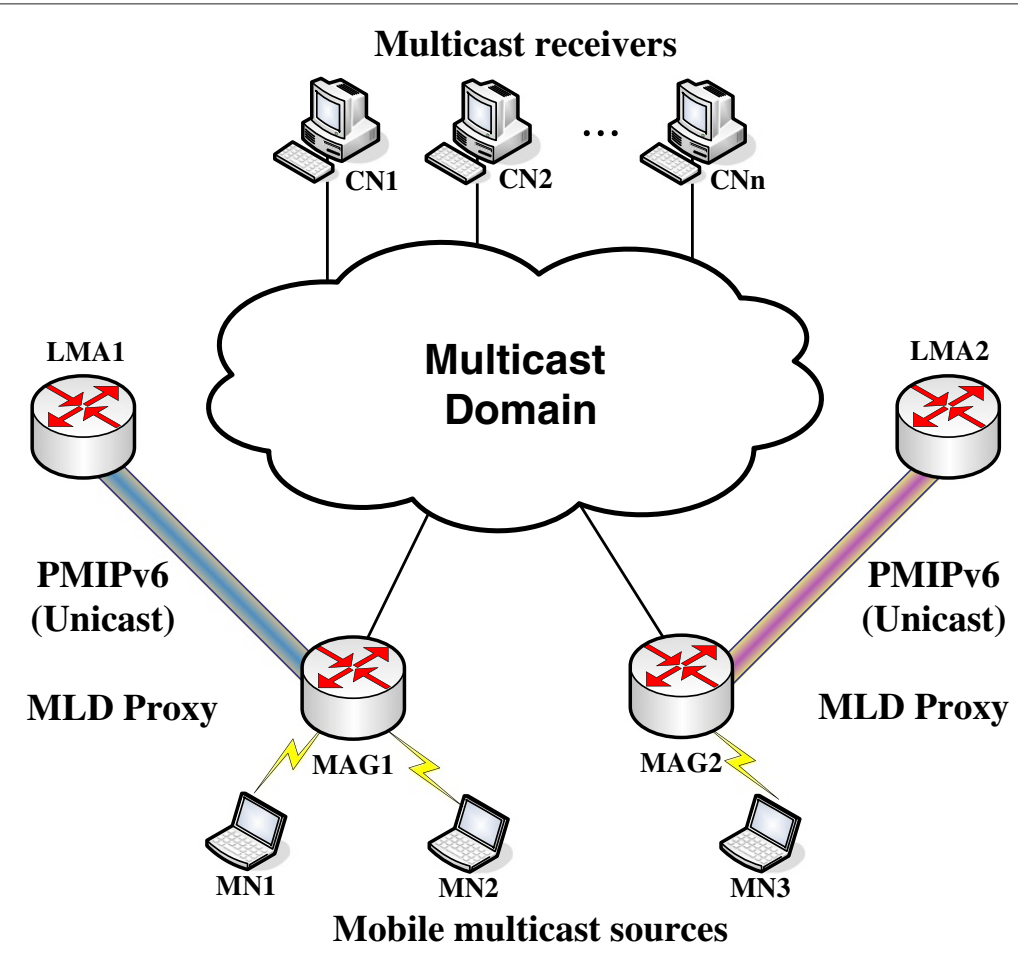

Figure 4 Deployment scenario of MLD proxies at the MAGs for the DMRS. 
to the multicast infrastructure. Especially when the MN moves to a place far away from the LMA, all the traffic must also be forwarded through this LMA and then the data flow will be routed through a very long path, which will cause a higher packet delivery cost and latency. In addition, there are deployment scenarios, where multicast services are available throughout the access network independent of the PMIPv6 routing system [37]. In these cases, the visited networks grant a local content distribution service with locally optimized traffic flows. Therefore, the DMRS for mobile multicast source support in PMIPv6 networks is proposed.

Direct multicast access can be supported by a multicast routing protocol such as PIM-SM, or by MLD proxies deployed at the MAGs, and the corresponding deployment scenarios are displayed in Figures 3 and 4, which separate multicast from PMIPv6 unicast routing. It is assumed that throughout these scenarios, all the MAGs (MLD proxies) are linked to a single multicast routing domain. In this way, when a mobile multicast source begins to send packets, its accessed MAG running multicast routing functions will seamlessly distribute these multicast traffic into a multicast cloud, or running a single proxy instance with up-link into the multicast domain will serve as a first-hop multicast gateway and avoid traffic duplication or detour routing. Whether the PIM-SM or MLD proxy is deployed at the MAG in the DMRS, the routing inefficiency problem proposed in the BS can all be avoided and both of them can provide a locally optimized routing.

\subsubsection{PIM-SM at the MAGs}

In the any source multicast (ASM) case, the MAG1, acting as a PIM DR, will encapsulate the packets originated by the multicast source $S$ (MN1) to the RP through the SR at first. The RP will then decapsulate and forward the packets down the RP-based distribution tree towards the receivers. After receiving the SR packets, the RP will initiate a source-specific join for creating a SPT to the mobile source $\mathrm{S}$ and issue a source register stop at the native arrival of data from S. Since the LMA1 is the MN1's topological anchor point in the PMIPv6 network, the $(S, G)$ tree will proceed from the RP via the LMA1 and then the LMA1-MAG1 tunnel to the mobile source MN1. Therefore, the RPs should be configured not to initiate (S, G) SPTs for mobile sources and thus remain in the SR case all the time. While in the SSM case, the established SPT will also firstly go through the LMA1 and then to the MAG1, in which the detour routing is introduced again just as same as the BS in the SPT case. Therefore, some extensions or solutions need to be proposed to improve the DMRS in the SSM case, which will be our future research work.
On the handover from the MAG1 to the MAG2, the point-to-point link between the mobile source MN1 and the MAG1 will go down, and all (S, ") flows terminate. Only when the MN1 reattaches to the MAG2 and completes the registration to the LMA1, it can transmit multicast packets again. Since the MAG2 takes place of the MAG1 to be the DR, the mobile source is treated as a new source at its new DR (MAG2). The MAG2 then immediately initiates the SR encapsulation to the RP, and (S, G) data continue to flow natively down the (*, G) RP-based tree.

\subsubsection{MLD proxies at the MAGs}

Multicast data submitted by the mobile source MN1 will reach the MLD proxy at the MAG1 that subsequently forwards flows to the upstream interface and also the downstream interface for the MN2 with appropriate subscription. Traversing the upstream will lead the traffic into the multicast infrastructure (e.g., to a PIM DR) which will route packets to all local MAGs that have joined the group, as well as further upstream according to the multicast protocol procedures and forwarding states. Besides, since a multicast source transmitting data via an MLD proxy is not directly connected to a PIM DR, the DR should also act as a PIM border router and activate the border-bit or run the BIDIRPIM as described in Section 3.1.3 to address this issue.

On the handover from the MAG1 to the MAG2, the mobile source MN1 will reattach at the MAG2 and can continue to send multicast packets as soon as PMIPv6 unicast configurations have been completed. Like at the MAG1, the new MLD proxy at the MAG2 will forward data to the upstream and downstream receivers. Receivers local to the MAG1, such as the MN2, will continue to receive group traffic via the local multicast distribution infrastructure following aggregated receiver reports of the previous proxy at the MAG1.

\section{Performance evaluation}

This section evaluates the signaling cost performance of the BS and the DMRS based on the PIM-SM at the MAGs and also compares with the MIP-BT and the MIP-RS schemes [3]. In this paper, the signaling cost is defined as the product of signaling message size and hop distance, and the unit is in bytes $\times$ hops [38]. Signaling cost has two major components: (i) signaling cost related to the mobility management and (ii) signaling cost related to the packet transmission [39]. We analyze the signaling cost of these schemes for both the above components through mathematical analysis. 




\subsection{Analytical model}

Figure 5 shows the analytical mobility model, which is an hexagonal cellular network architecture. It is assumed that the PMIPv6 domain consists of $R$ rings. Each $\operatorname{ring} r(r \geq 0)$ is composed of $6 r$ cells and each cell represents an MAG subnet. Therefore, the number of MAG subnets up to ring $R, N(R)$, is calculated using the following equation:

$$
N(R)=\sum_{i=1}^{R} 6 r+1=3 R(R+1)+1
$$

\subsection{Mobility model}

In terms of user mobility model, because the fluid-flow model is widely used to analyze cell boundary crossingrelated issues, such as handoff, in legacy wireless networks where voice is the dominant service $[40,41]$, we adopt this model to analyze the signaling cost in the PMIPv6 network for simplicity. Under the fluid-flow model, the direction of an MN's movement in a PMIPv6 domain is uniformly distributed over $[0,2 \pi]$ at an average velocity of $v$, and the MNs are uniformly distributed with density $\rho$. We assume that the perimeter of a cell is $l_{c}$, and hence based on the earlier description in Section 4.1 the perimeter of the PMIPv6 domain consisting of $R$ rings, denoted as $L(R)$, can be obtained using the following equation [42]:

$$
L(R)=6 \times(2 R+1) \times \frac{l_{c}}{6}(R \geq 1) .
$$

Let $R_{\mathrm{c}}$ and $R_{\mathrm{d}}$ be cell boundary crossing rate and PMIPv6 domain crossing rate, respectively. Therefore, the cell and domain boundary crossing rates are as follows [43]:

$$
\begin{aligned}
& R_{\mathrm{c}}=\frac{\rho \cdot v \cdot l_{\mathrm{c}}}{\pi} \\
& R_{\mathrm{d}}=\frac{\rho \cdot v \cdot L(R)}{\pi}
\end{aligned}
$$

\subsection{Signaling cost analysis}

The location update cost and the packet delivery cost are denoted by $C_{\mathrm{LU}}$ and $C_{\mathrm{PD}}$, respectively. Then, the total cost is the sum of location update cost and packet delivery cost, which is as follows:

$$
C_{\mathrm{TOT}}=C_{\mathrm{LU}}+C_{\mathrm{PD}}
$$

\subsubsection{Location update cost}

An MN moves either within a local PMIPv6 domain or out of a PMIPv6 domain. Therefore, an MN performs two types of binding update: the global binding update and the local binding update. When an MN moves between different PMIPv6 domains, the global binding update is performed in which an $\mathrm{MN}$ registers its new location with the LMA and the HA. On the other hand, if an MN moves within a local PMIPv6 domain, the local binding update is performed in which the MN only needs to register its new location with the LMA. Let $C_{\mathrm{g}}$ and $C_{\mathrm{l}}$ denote the signaling cost in the global binding update and the local binding update, respectively. Since the signaling cost is proportional to the distance of two network entities in the IP networks [42], for basic PMIPv6, $C_{\mathrm{g}}$ and $C_{\mathrm{l}}$ can be obtained from the below equations:

$$
\begin{aligned}
& C_{\mathrm{g}}=(\mathrm{pbu}+\mathrm{pba}) \cdot \tau \cdot d_{\mathrm{lm}}+(\mathrm{bu}+\mathrm{ba}) \cdot\left(\kappa+\tau \cdot d_{\mathrm{hm}}\right) \\
&+\mathrm{pc}_{\mathrm{h}}+\mathrm{pc}_{\mathrm{m}}+\mathrm{pc}_{\mathrm{l}} \\
& C_{\mathrm{l}}=(\mathrm{pbu}+\mathrm{pba}) \cdot \tau \cdot d_{\mathrm{lm}}+\mathrm{pc}_{\mathrm{m}}+\mathrm{pc}_{\mathrm{l}},
\end{aligned}
$$

where $\kappa$ and $\tau$ are the unit transmission costs in a wireless and a wired link, respectively. Let $d_{\mathrm{lm}}$, and $d_{\mathrm{hm}}$ be the hop distance between the LMA and the MAG, and between the HA and the MAG, respectively. And $d_{\mathrm{hm}}=d_{\mathrm{hl}}+d_{\mathrm{lm}}$, where $d_{\mathrm{hl}}$ is the hop distance between the HA and the LMA. pbu, pba, bu, and ba are the sizes of the signaling messages for the location update in PMIPv6 and MIPv6, respectively. $\mathrm{pc}_{\mathrm{h}}, \mathrm{pc}_{\mathrm{m}}$, and $\mathrm{pc}_{\mathrm{l}}$ are the processing costs for binding update procedures at the HA, the MAG, and the LMA, respectively. 
According to the analysis in Section 4.2, we can obtain the location update cost per MN as follows:

$$
C_{\mathrm{LU}}=\frac{R_{\mathrm{d}} \cdot C_{\mathrm{g}}+\left(N_{\mathrm{m}} \cdot R_{\mathrm{c}}-R_{\mathrm{d}}\right) \cdot C_{\mathrm{l}}}{\rho \cdot A(R)},
$$

where $N_{\mathrm{m}}$ is the number of the MAGs in a PMIPv6 domain, and $A(R)$ refers to the area of a PMIPv6 domain and can be obtained as $A(R)=A_{\mathrm{c}} \cdot N_{\mathrm{m}}=\frac{\sqrt{3}}{24} \cdot l_{\mathrm{c}}^{2} \cdot N_{\mathrm{m}}$, where $A_{\mathrm{c}}$ is the area of a cell.

Since the binding update procedure of the BS is just the same as that of the DMRS, the location update cost for the $\mathrm{BS}\left(C_{\mathrm{LU}}^{\mathrm{BS}}\right)$ is equal to that for the DMRS $\left(C_{\mathrm{LU}}^{\mathrm{DMRS}}\right)$, that is,

$$
C_{\mathrm{LU}}^{\mathrm{BS}}=C_{\mathrm{LU}}^{\mathrm{DMRS}}=C_{\mathrm{LU}}
$$

Similarly, we can obtain the location update cost for the MIP-BT and the MIP-RS as follows:

$$
C_{\mathrm{LU}}^{\mathrm{MIP}-\mathrm{BT}}=C_{\mathrm{LU}}^{\mathrm{MIP}-\mathrm{RS}}=\frac{N_{\mathrm{m}} \cdot R_{\mathrm{c}} \cdot C_{\mathrm{h}}}{\rho \cdot A(R)},
$$

where $C_{\mathrm{h}}$ is the binding update signaling cost to the HA for an MN in MIPv6 networks, and $C_{\mathrm{h}}$ can be obtained from the below equations:

$$
C_{\mathrm{h}}=(\mathrm{bu}+\mathrm{ba}) \cdot\left(\kappa+\tau \cdot d_{\mathrm{hm}}\right)+\mathrm{pc}_{\mathrm{mn}}+\mathrm{pc}_{\mathrm{h}},
$$

where $\mathrm{pc}_{\mathrm{mn}}$ is the processing cost for binding update procedure at the $\mathrm{MN}$.

\subsubsection{Packet delivery cost}

Based on the description in Section 3.2, we choose the ASM case to analyze the packet delivery cost for the BS and the DMRS. Under the movement of the multicast source in the ASM case, the packet transmission from the RP to the receivers for both of the solutions keeps unchanged and is no difference from each other; therefore, in this section, we just consider the packet delivery cost from the mobile multicast source $\mathrm{S}$ to the RP.
The cost for packet delivery procedure $\left(C_{\mathrm{PD}}\right)$ can be expressed as [42,44-46]:

$$
C_{\mathrm{PD}}=T_{S-\mathrm{RP}}+P_{\mathrm{m}}+P_{\mathrm{l}},
$$

where $T_{S-\mathrm{RP}}$ denotes the transmission cost of packet delivery from the mobile multicast source to the RP. $P_{\mathrm{m}}$ and $P_{1}$ denote the processing costs of packet delivery at the MAG and the LMA, respectively.

Figure 6 shows the packet delivery procedures for the $\mathrm{BS}$ and the DMRS that are varying with the MAG residence time. As can be seen from Figure 6, in the BS, the packet transmission from the LMA to the RP adopts the SPT instead of the SR after $t_{1}$, while in the DMRS, the packet transmission from the MAG to the RP keeps the SR unchanged all the time.

Therefore, the transmission cost of packet delivery from the mobile multicast source to the RP in the BS can be divided into the SR and SPT case and can be calculated as follows:

$$
\begin{aligned}
& T_{S-\mathrm{RP}}^{\mathrm{BS}-\mathrm{SR}}=m \cdot \lambda_{\mathrm{s}} \cdot S \cdot L_{\mathrm{s}} \cdot \kappa+m \cdot \lambda_{\mathrm{s}} \cdot S \cdot L_{\mathrm{s}} \\
& \cdot\left(1+\frac{S_{\mathrm{h}}}{L_{\mathrm{s}}}\right) \cdot\left(d_{\mathrm{lm}} \cdot \tau+d_{\mathrm{lr}} \cdot \tau\right) \quad\left(0 \leq t \leq t_{1}\right)
\end{aligned}
$$

$$
\begin{array}{r}
T_{S-\mathrm{RP}}^{\mathrm{BS}-\mathrm{SPT}}=m \cdot \lambda_{\mathrm{s}} \cdot S \cdot L_{\mathrm{s}} \cdot \kappa+m \cdot \lambda_{\mathrm{s}} \cdot S \cdot L_{\mathrm{s}} \cdot\left(1+\frac{S_{\mathrm{h}}}{L_{\mathrm{s}}}\right) \\
\cdot d_{\mathrm{lm}} \cdot \tau+m \cdot \lambda_{\mathrm{s}} \cdot S \cdot L_{\mathrm{s}} \cdot d_{\mathrm{lr}} \cdot \tau \quad\left(t>t_{1}\right),
\end{array}
$$

where $t$ is the MAG residence time for a mobile multicast source, $d_{\text {lr }}$ denotes the hop distance between the LMA and the RP, $\lambda_{\mathrm{s}}$ denotes the multicast source session arrival rate, $S$ represents the average session size in the unit of packet, $L_{\mathrm{s}}$ is the multicast packet size, $S_{\mathrm{h}}$ is the size of the extra header for the packet encapsulation, and $m$ represents the number of multicast groups for a certain source.

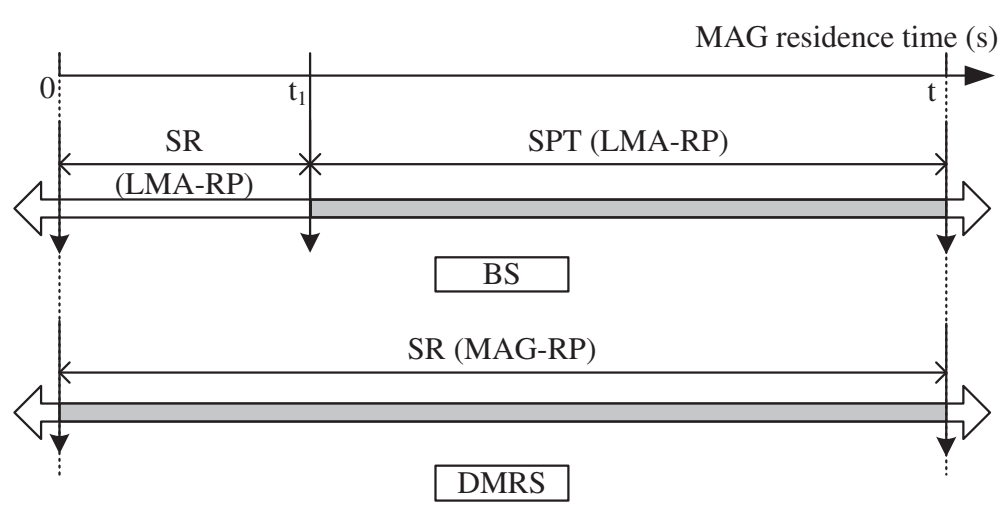

Figure 6 Packet delivery procedures for the BS and the DMRS varying with the MAG residence time. 
However, because the packets are always delivered from the MAG to the RP through the SR in the DMRS, the transmission cost of packet delivery from the mobile multicast source to the RP in the DMRS can be calculated as

$$
T_{S-\mathrm{RP}}^{\mathrm{DMRS}}=m \cdot \lambda_{\mathrm{s}} \cdot S \cdot L_{\mathrm{s}} \cdot\left[\kappa+\left(1+\frac{S_{\mathrm{h}}}{L_{\mathrm{s}}}\right) \cdot d_{\mathrm{mr}} \cdot \tau\right] \quad(t \geq 0)
$$

where $d_{\mathrm{mr}}$ is the hop distance between the MAG and the RP.

In the BS, when a packet arrives at the MAG, the MAG selects the corresponding LMA for the multicast source $\mathrm{MN}$ from the mapping table and then the packet is routed to the LMA. Therefore, the processing cost of packet delivery at the MAG in the BS includes the lookup cost ( $\left.C_{\text {lookup }}\right)$ and the routing cost $\left(C_{\text {routing }}\right)$. The lookup cost is proportional to the size of the mapping table, and the size of the mapping table at the MAG is proportional to the number of MNs located in the coverage of this MAG. We assume that the average number of users located in the coverage of an MAG is $N_{\mathrm{mn}}$. According to the fluidflow model, $N_{\mathrm{mn}}$ can be given as: $N_{\mathrm{mn}}=\rho \cdot A_{\mathrm{c}}$. On the other hand, the routing cost is proportional to the logarithm of the length of the routing table [47], for IP routing table lookup is based on the longest prefix matching and most implementations use the traditional Patricia trie [48]. Therefore, the processing cost at the MAG in the BS can be expressed as [42,44-46]:

$$
\begin{aligned}
P_{\mathrm{m}}^{\mathrm{BS}} & =\lambda_{\mathrm{s}} \cdot S \cdot\left(C_{\text {lookup }}+C_{\text {routing }}\right) \\
& =\lambda_{\mathrm{s}} \cdot S \cdot\left[\alpha N_{\mathrm{mn}}+\beta \log \left(L_{\mathrm{m}}\right)\right]
\end{aligned}
$$

where $\alpha$ and $\beta$ are the weighting factors of mapping table and routing table lookups, respectively. $L_{\mathrm{m}}$ is the length of the routing table at the MAG. However, only the routing cost needs to be considered for the processing cost of packet delivery at the LMA in the BS. Therefore, the processing cost at the LMA in the BS can be expressed as:

$$
P_{1}^{\mathrm{BS}}=\lambda_{\mathrm{s}} \cdot S \cdot C_{\text {routing }}=\lambda_{\mathrm{s}} \cdot S \cdot\left[\beta \log \left(L_{1}\right)\right],
$$

where $L_{1}$ is the length of the routing table at the LMA.

In the DMRS, when a packet arrives at the MAG, the MAG directly transmits the multicast packets to the RP through the SR. Therefore, there is no processing cost at the LMA in the DMRS, and the processing cost of packet delivery at the MAG only contains the routing cost. Then the processing cost at the MAG in the DMRS can be calculated as follows:

$$
P_{\mathrm{m}}^{\mathrm{DMRS}}=\lambda_{\mathrm{s}} \cdot S \cdot C_{\text {routing }}=\lambda_{\mathrm{s}} \cdot S \cdot\left[\beta \log \left(L_{\mathrm{m}}\right)\right]
$$

Similarly, the transmission cost for the MIP-BT and the MIP-RS can be calculated as:
Table 2 Performance analysis parameters

\begin{tabular}{llll}
\hline Parameter & Value & Parameter & Value \\
\hline$\kappa$ & 2 & $\tau$ & 1 \\
pbu & 76 bytes & pba & 76 bytes \\
bu & 72 bytes & ba & 52 bytes \\
$\mathrm{pc}_{\mid}$ & 12 & $\mathrm{pc}_{\mathrm{m}}$ & 12 \\
$\mathrm{pc}_{\mathrm{h}}$ & 24 & $\mathrm{pc}_{\mathrm{mn}}$ & 12 \\
$d_{\mathrm{lm}}$ & 2 hops & $d_{\mathrm{lr}}$ & 4 hops \\
$d_{\mathrm{hl}}$ & 6 hops & $d_{\mathrm{hr}}$ & 4 hops \\
$S$ & 10 & $S_{\mathrm{h}}$ & 40 bytes \\
$m$ & $1 \sim 30$ & $L_{\mathrm{s}}$ & 1,500 bytes \\
$I_{c}$ & $120 \mathrm{~m}$ & $\lambda_{\mathrm{s}}$ & 0.1 \\
$\rho$ & 0.0002 & $V$ & $20 \mathrm{~m} / \mathrm{s}$ \\
$\alpha$ & 0.1 & $\beta$ & 0.2 \\
\hline
\end{tabular}

$$
\begin{aligned}
T_{S-\mathrm{RP}}^{\mathrm{MIP}-\mathrm{BT}-\mathrm{SR}}=m & \cdot \lambda_{\mathrm{s}} \cdot S \cdot L_{\mathrm{s}} \cdot\left(1+\frac{S_{\mathrm{h}}}{L_{\mathrm{s}}}\right) \\
& \cdot\left(\kappa+d_{\mathrm{hm}} \cdot \tau+d_{\mathrm{hr}} \cdot \tau\right) \quad\left(0 \leq t \leq t_{1}\right)
\end{aligned}
$$

$$
\begin{aligned}
T_{S-\mathrm{RP}}^{\mathrm{MIP}-\mathrm{BT}-\mathrm{SPT}}= & m \cdot \lambda_{\mathrm{s}} \cdot S \cdot L_{\mathrm{s}} \cdot\left(1+\frac{S_{\mathrm{h}}}{L_{\mathrm{s}}}\right) \cdot\left(\kappa+d_{\mathrm{hm}} \cdot \tau\right) \\
& +m \cdot \lambda_{\mathrm{s}} \cdot S \cdot L_{\mathrm{s}} \cdot d_{\mathrm{hr}} \cdot \tau \quad\left(t>t_{1}\right) \quad(20)
\end{aligned}
$$

$$
T_{S-\mathrm{RP}}^{\mathrm{MIP}-\mathrm{RS}}=m \cdot \lambda_{\mathrm{s}} \cdot S \cdot L_{\mathrm{s}} \cdot\left[\kappa+\left(1+\frac{S_{\mathrm{h}}}{L_{\mathrm{s}}}\right) \cdot d_{\mathrm{mr}} \cdot \tau\right] \quad(t \geq 0)
$$

where $d_{\text {hr }}$ represents the hop distance between the HA and RP.

\subsection{Numerical results}

This subsection presents various analysis results based on the developed analytical mobility model. Table 2 shows the parameter values for the analysis, which are referenced from $[42-46,49]$.

\subsubsection{Location update cost vs. user mobility}

Figure 7 depicts the variation in the location update cost for the BS, DMRS, MIP-BT, and MIP-RS schemes as the MN's average velocity is changed. As shown in Figure 7, all the location update costs increase with the increased MN's average velocity, as an MN will have a higher domain crossing rate under a higher average velocity, thereby a higher location update cost will be required. In addition, the BS and DMRS have lower location update cost than the MIP-BT and MIP-RS, since PMIPv6 does not require any participation of the $\mathrm{MN}$ in mobility-related signaling 




Figure 7 Comparison of location update cost under the average velocity.

[50]. Besides, the location update cost of a ring size of 4 $(R=4)$ is smaller than that of a ring size of $1(R=1)$ in the BS and DMRS, and the signaling gain defined as the location update cost at $R=4$ to that of $R=1$ is $56.7 \%$. The reason is that an MN located in the PMIPv6 domain with a large ring size is more inclined to perform local binding update procedures. While the ring size has no effects on the location update cost for the MIP-BT and MIP-RS, for MIPv6 is a global mobility management protocol.

\subsubsection{Packet delivery cost vs. hop distance between the MAG and RP}

Figure 8 shows the impact of the hop distance between the MAG and RP on the packet delivery cost. Since we set the hop distance between the LMA/HA and MAG and between the LMA/HA and RP as fixed values, we observe in Figure 8 that all the results for the BS and

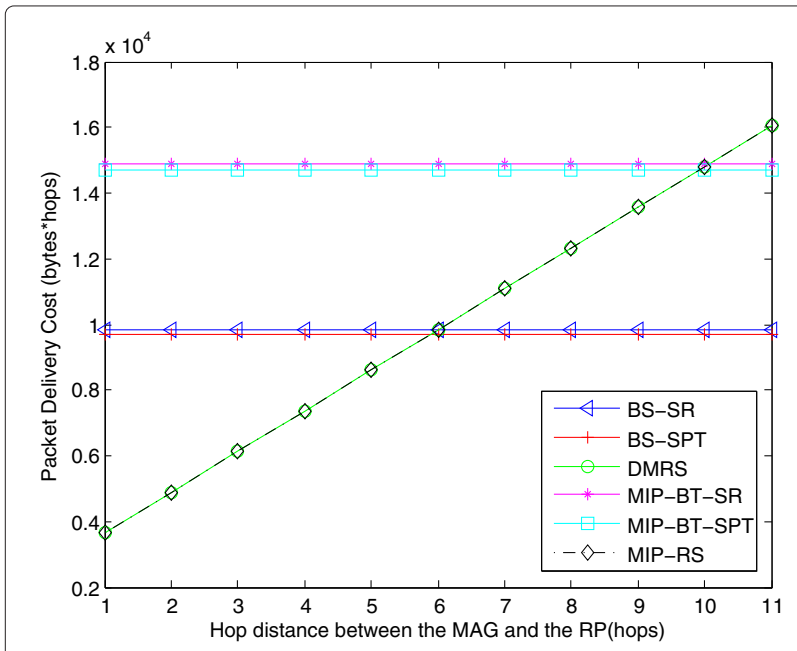

Figure 8 Comparison of packet delivery cost under the distance between the MAG and RP.
MIP-BT remain unchanged and the packet delivery costs for the DMRS and MIP-RS increase with the increased hop distance between the MAG and RP. The reason is that shorter hop distance between the MAG and RP will bring about more optimized routing for the DMRS and MIP-RS and then less signaling cost is required. Therefore, when the routing for the DMRS/MIP-RS is more optimized than the BS/MIP-BT, the packet delivery cost of the DMRS/MIP-RS is lower than that of the BS/MIP-BT. Since the DR is always the LMA/HA in the BS/MIP$\mathrm{BT}$, the multicast tree needs not to be reestablished on the handover, while the reconstruction of the tree is needed in the DMRS/MIP-RS for the DR is changed. Consequently, with the increased distance between the MAG and RP, the packet delivery cost of the DMRS/MIPRS gradually increases and finally exceeds the cost of the BS/MIP-BT in the SR case and the SPT case when the hop distances are 6/10 and 5.9/9.9, respectively. In addition, the packet delivery cost of the BS/MIP-BT in the SR case is higher than that of the BS/MIP-BT in the SPT case, for the BS/MIP-BT in the SPT case transmits data via the SPT rather than the SR tunnel. Besides, the BS has lower packet delivery cost than the MIP-BT, and the packet delivery cost of the DMRS is equal to that of the MIP-RS.

\subsubsection{Packet delivery cost vs. number of multicast groups}

Figure 9 compares the results of packet delivery cost under the number of multicast groups for a certain source. From Figure 9, we observe that all the packet delivery costs increase with the increased number of multicast groups because more groups for a certain multicast source need more packet transmission. Since MIPv6 is a host-based mobility protocol and PMIPv6 is a network-based mobility protocol, all the packet delivery costs of the MIP-BT



Figure 9 Comparison of packet delivery cost under the number of multicast groups for a certain source. 


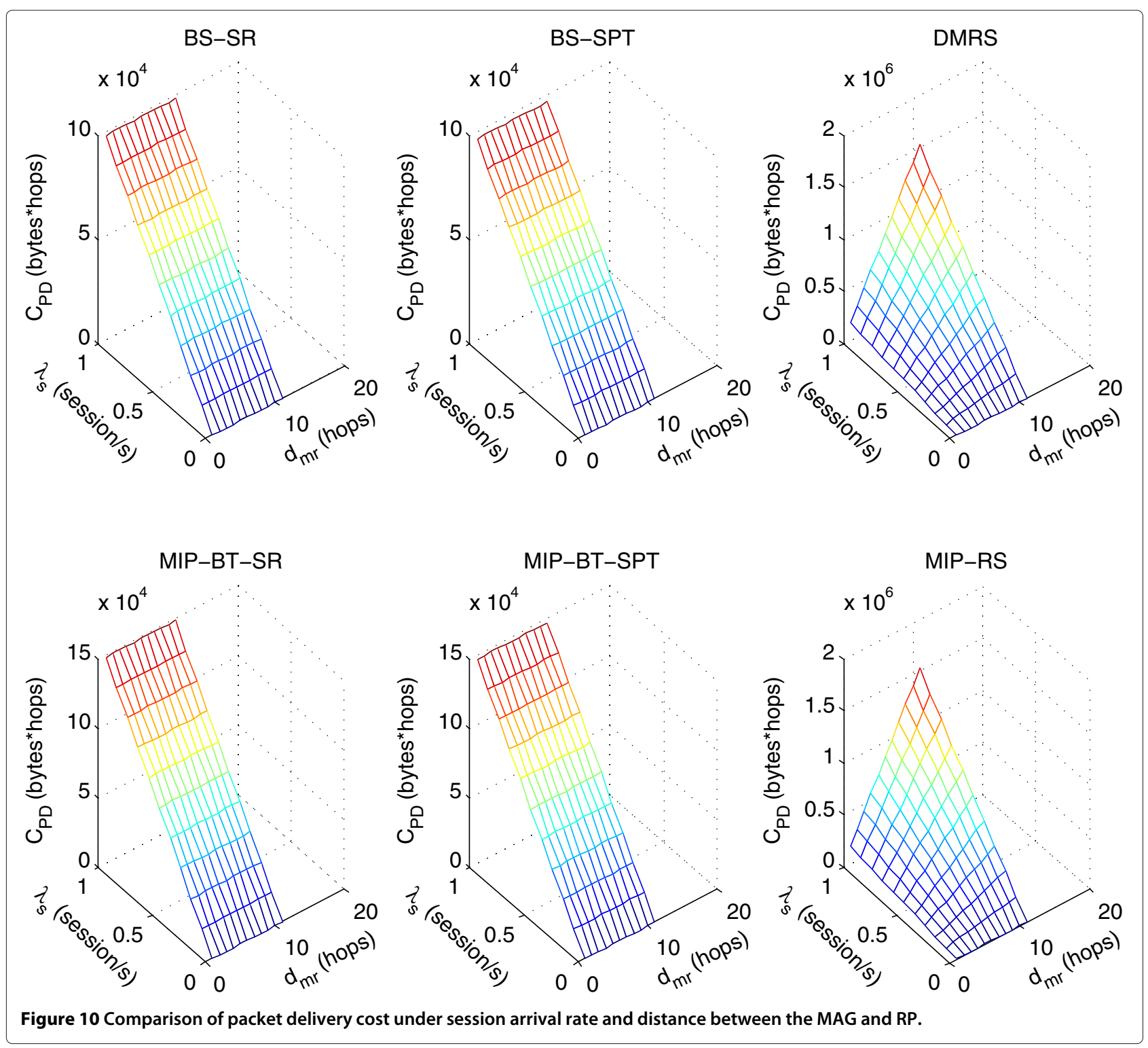

are higher than those of the BS. Besides, the MIP-BT and the BS in the SPT case have lower packet delivery cost than those in the SR case for the SR encapsulation will lead to more delivery cost than the SPT. While the MIP-RS has the same packet delivery cost with the DMRS, the reason is that they all transmit data directly to the RP through the SR by the DR.

\subsubsection{Packet delivery cost vs. session arrival rate and hop distance between the MAG and RP}

Figure 10 presents the packet delivery cost with the impact of the session arrival rate and the hop distance between the MAG and RP. In Figure 10, all the packet delivery costs increase with the increased session arrival rate, for more packets should be transmitted in this case. At the same time, all the packet delivery costs for the DMRS and MIP-RS increase with the increased hop distance between the MAG and RP, while the results for the BS and MIP-BT remain unchanged, which is the same with Figure 8. Meanwhile, the packet delivery cost of the BS is lower than that of the MIP-BT, and the packet delivery cost of the DMRS is equal to that of the MIP-RS. Besides, the packet delivery cost of the BS/MIP-BT in the SPT case is lower than that of the BS/MIP-BT in the SR case.

\subsubsection{Total signaling cost vs. user mobility}

In Figure 11, we make a comparison among MIP-BT, MIP$\mathrm{RS}, \mathrm{BS}$, and DMRS to evaluate the total signaling cost under the average velocity. As illustrated in Figure 11, all the total costs decline with the decreased average velocity, for an $\mathrm{MN}$ will have a lower domain crossing rate under 


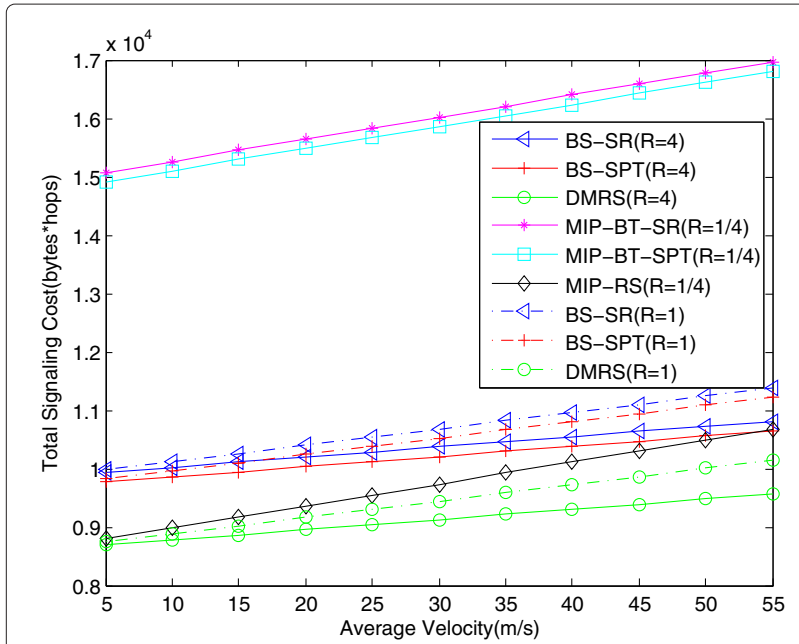

Figure 11 Comparison of total signaling cost under the average velocity.

a smaller average velocity. Meanwhile, the total costs in the MIP-BT and MIP-RS are higher than the BS and DMRS, and the cost of the BS/MIP-BT in the SR case is higher than that of the BS/MIP-BT in the SPT case and higher than that of the DMRS/MIP-RS. The reason is that the BS/MIP-BT in the SPT case adopts the SPT to transfer data instead of the SR encapsulation, and the DMRS/MIP-RS has more optimized routing than the BS/MIP-BT in the SR case. Besides, since the hop distance between the MAG and RP is set as a fixed value 5 , which is more optimized than the path length of the MIP-BT/BS in the SPT case, the total cost of the MIPRS/DMRS is lower than that of the MIP-BT/BS in the SPT case. In addition, all the total costs for the BS and DMRS at $R=1$ are higher than those at $R=4$, for an



Figure 12 Comparison of total cost under the distance between the MAG and RP.



MN located in the PMIPv6 domain with a small ring size is more likely to perform global binding update procedures. However, the value of $R$ has no effects on the total costs for the MIP-BT and MIP-RS, for the MN always performs global binding update procedure on handover in MIPv6 networks.

\subsubsection{Total signaling cost vs. hop distance between the MAG and $R P$}

Figure 12 compares the results of total signaling cost under the hop distance between the MAG and RP. The overall trends in Figure 12 are almost the same with Figure 8. However, there is a big difference between the two figures, that is, the total cost of the MIP-RS is higher than that of the DMRS, while the packet delivery cost of

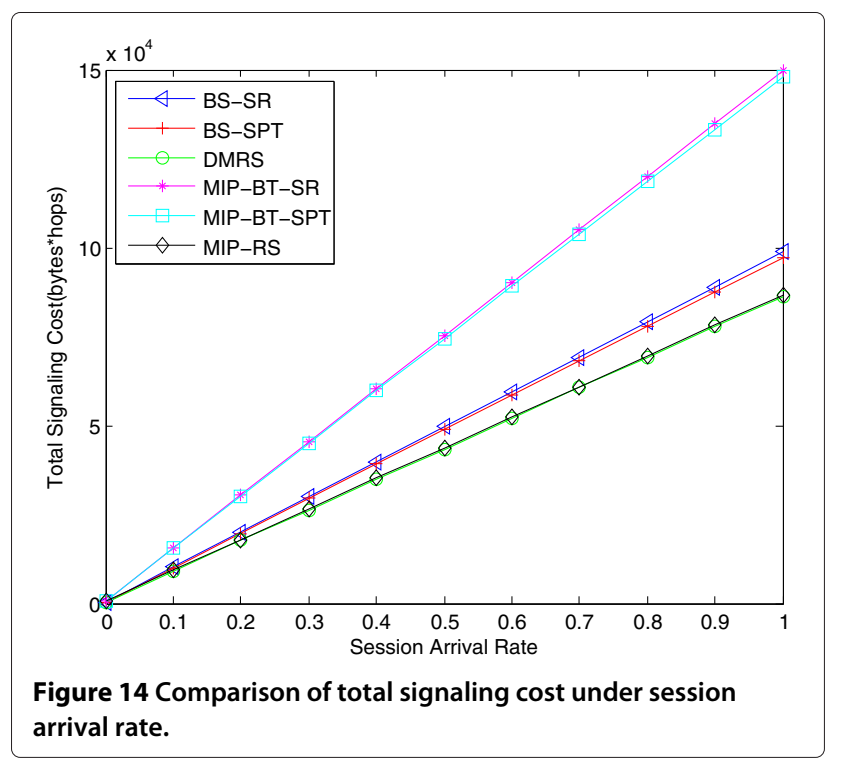






Figure 15 Comparison of signaling costs under the optimal PMIPv6 domain size for the static MN.

the MIP-RS is equal to that of the DMRS under the same situation. The reason is that the total signaling cost consists of the packet delivery cost and the location update cost, and the location update cost of the MIP-RS is higher than that of the DMRS.

\subsubsection{Total signaling cost vs. number of multicast groups}

The results of total signaling cost under the number of multicast groups for a certain source are shown in Figure 13. It can be observed in Figure 13 that all the total costs increase with the increased number of multicast groups. The overall trends in Figure 13 are almost the same with Figure 9. The difference is that the packet delivery cost of the DMRS is equal to that of the MIPRS, while the total cost of the DMRS is lower than that



Figure 16 Comparison of signaling costs under the optimal PMIPv6 domain size for the dynamic MN. of the MIP-RS under the situation of the same number of groups.

\subsubsection{Total signaling cost vs. session arrival rate}

The impact of the session arrival rate on the total signaling cost is presented in Figure 14. As illustrated in Figure 14, all the total costs increase with the increased session arrival rate, for more packets should be transmitted in this case. Besides, the total costs in the BS and DMRS are lower than the MIP-BT and MIP-RS. Meanwhile, due to the handover from the SR to the SPT between the LMA/HA and RP and also more optimized path for the DMRS and MIP-RS, the total cost of the BS/MIP$\mathrm{BT}$ in the SR case is higher than that of the BS/MIP$\mathrm{BT}$ in the SPT case and also higher than that of the DMRS/MIP-RS.

\subsubsection{Total signaling cost vs. optimal PMIPv6 domain size}

To minimize the total signaling cost, it is important to determine the optimal PMIPv6 domain size for network deployment. When determining the optimal PMIPv6 size, the tradeoff relationship between the location update cost and the packet delivery cost needs to be taken into account. The optimal PMIPv6 domain size is examined with the impact of different MNs, and different parameter values are used to describe an MN's mobility. Based on the fluid-flow model, the average velocities of static MNs and dynamic MNs are 20 and $200 \mathrm{~m} / \mathrm{s}$, respectively. Additionally, the user density is 0.0002 [42].

Figures 15 and 16 illustrate the total signaling cost, the location update cost, and the packet delivery cost of the BS and the DMRS for the static MN and dynamic $\mathrm{MN}$, respectively. In the case of the static $\mathrm{MN}$, all the packet delivery costs are much higher than the location update costs for all PMIPv6 domain sizes, which means that the packet delivery cost is the primary factor for the total signaling cost. As shown in Figure 15, the packet delivery cost increases and the location update cost decreases with the increased PMIPv6 domain size; consequently, the optimal PMIPv6 domain size is 0 when minimizing the packet delivery cost. Therefore, a small PMIPv6 domain has the advantage of reducing the total signaling cost of the BS and the DMRS in the case of the static MN. However, in the case of the dynamic MN, the location update cost occupies the dominant parts for the total signaling cost especially for smaller PMIPv6 domain sizes and decreases as the PMIPv6 domain size increases. As presented in Figure 16, when the ring size is 10 , the location update cost is the lowest and then the total signaling cost is also the minimum. Therefore, a large PMIPv6 domain has the benefit for reducing the total signaling cost of the BS and the DMRS in the case of the dynamic MN. Thus, the above analysis results indicate 


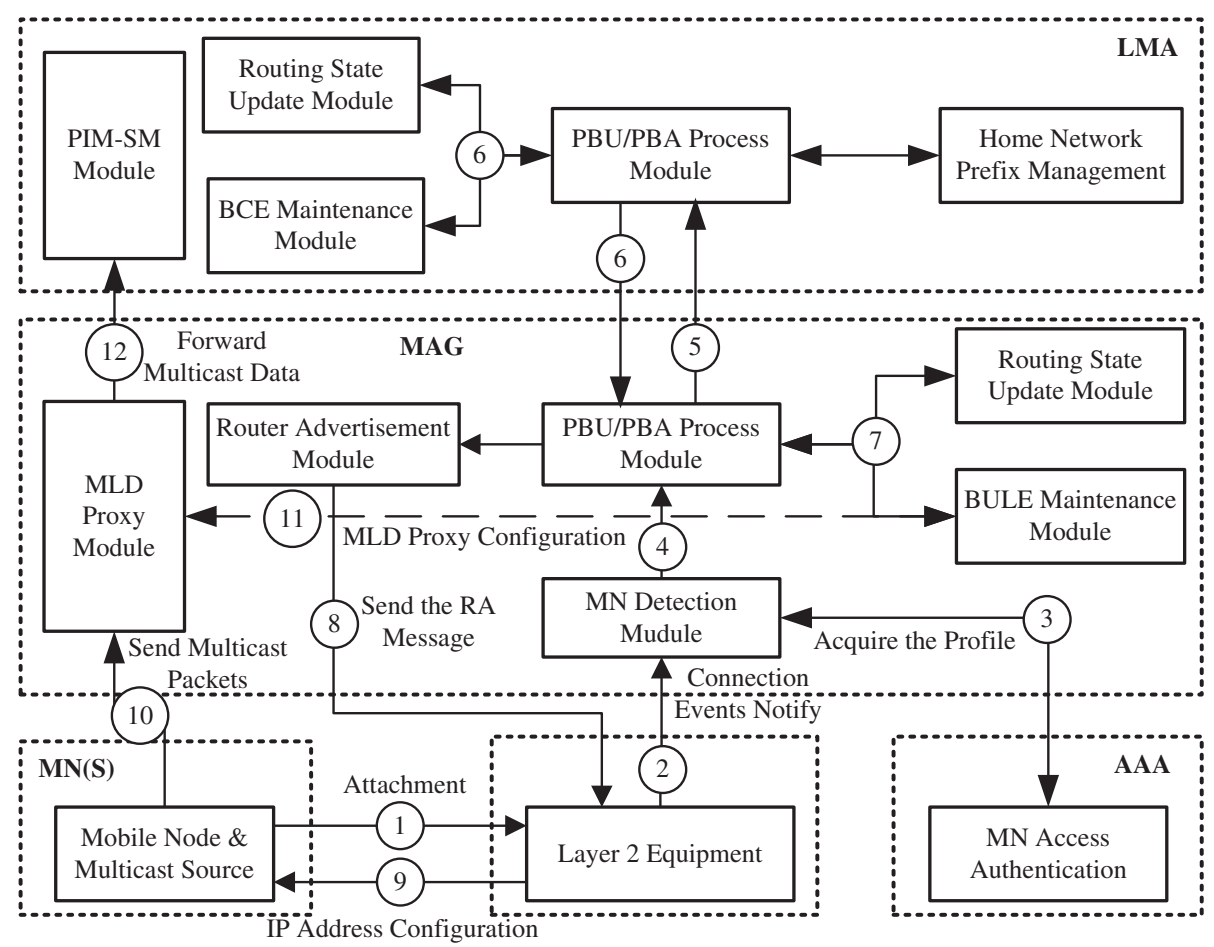

Figure 17 Implementation modules for mobile multicast source support in PMIPv6 networks.

that as the user mobility increases, the optimal PMIPv6 domain size increases.

\section{Experimental system development}

In this section, we present and analyze the results of our experimental evaluation of the proposed schemes, focusing on the performance of the multicast disruption time and the packet loss on handover.

\subsection{Implementation overview}

Figure 17 presents the whole BS implementation framework. The implementation covers three basic parts - the PMIPv6, MLD proxy, and PIM-SM protocols, where the PMIPv6 is based on the MIPL2.0 (MIPv6 for Linux; Helsinki University of Technology, Helsinki, Finland) [51] and MLD proxy is based on mcproxy (multicast proxy for IGMP/MLD; Hamburg University of Applied Sciences, Hamburg, Germany) [52]. The detailed PMIPv6 implementation module is shown in Figure 17, while the MLD proxy module is illustrated in Figure 18.

As shown in Figure 17, the detailed operation flow of mobile multicast source support in the PMIPv6 network is described as follows:

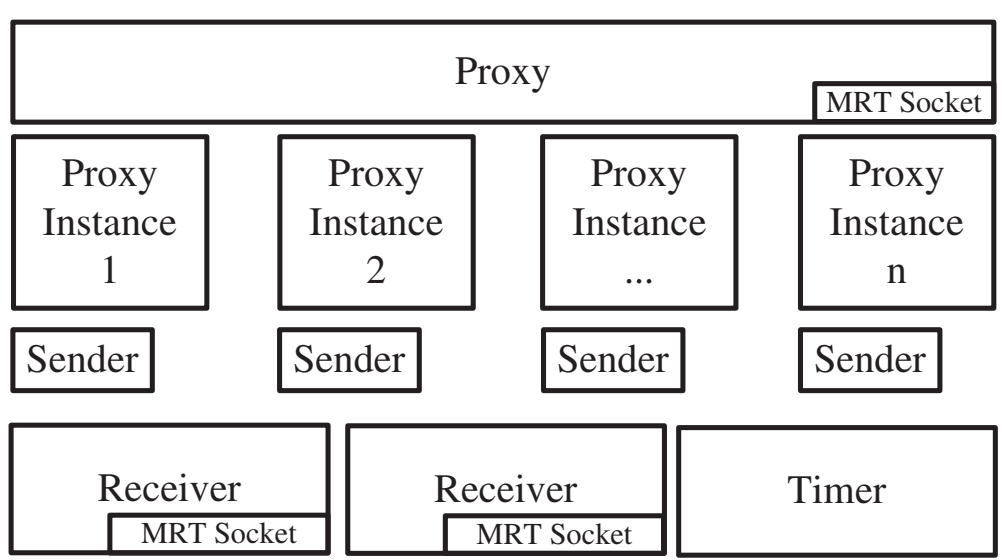

Figure 18 Mcproxy module. 


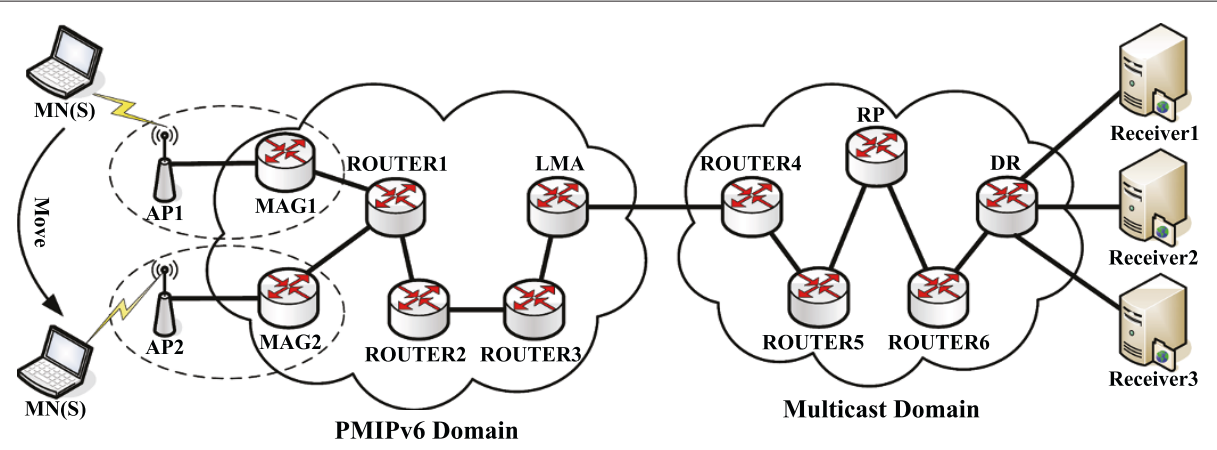

Figure 19 The experimental test-bed topology.

1. At first, an $\mathrm{MN}$ attaches to the layer 2 equipment (Cisco 1200 AP; Cisco Systems Inc., San Jose, CA, USA).

2. The Cisco 1200 AP firstly detects the attachment event and then notifies this event to the MAG.

3. The MAG gets the MN's layer 2 address from the notified message and acquires the $\mathrm{MN}$-identifier and policy profile from the policy server. For simplicity, the profile is stored in the local store for our implementation.

4. The MAG gets the MN-identifier and AAA information.

5. The MAG sends the proxy binding update (PBU) message to the LMA.

6. After receiving the PBU message, the LMA updates the routing states and maintains binding caches and then replies with a proxy binding acknowledgement (PBA) message to the MAG.

7. Just like the LMA, the MAG performs the PBU/PBA module to maintain the binding update lists and update the routing states.

8. After finishing the registration with the LMA, the MAG sends the router advertisement (RA) message

Table 3 Implementations configuration

\begin{tabular}{ll}
\hline Network entity & Configuration/IPv6 address \\
\hline LMA & NEL NGIID HA 2600/2601 \\
MAG & NEL NGIID MA 2600/2601 \\
Access point & Cisco 1200 AP \\
DR & NEL NGIID WR 2600/2601 \\
RP & NEL NGIID WR 2600/2601 \\
ROUTER & NEL NGIID A3600 \\
LMA & $3 f f e: 3240: 8007: 2005:: \mathrm{b} / 64$ \\
MAG1 & $3 f f e: 3240: 8007: 2003:: \mathrm{a} / 64$ \\
MAG2 & $3 f f e: 3240: 8007: 2001:: \mathrm{a} / 64$ \\
DR & $3 f f e: 3240: 8007: 2007:: \mathrm{b} / 64$ \\
RP & $3 f f e: 3240: 8007: 2006:: \mathrm{b} / 64$ \\
\hline
\end{tabular}

to advertise the home network prefix (HNP) for the MN.

9. Receiving the RA message containing the HNP, the MN configures its IP address based on this HNP.

10. As the multicast source, the MN sends multicast packets to the MAG running the MLD proxy.

11. The MAG configures the upstream interface of its MLD proxy at the tunnel towards the MN's corresponding LMA.

12. The multicast packets are forwarded through the PMIPv6 bidirectional tunnel to the LMA running the PIM-SM protocol.

\subsection{Experimental setup}

Figure 19 displays our experimental test-bed topology which consists of one multicast source $M N$, one DR, one RP, one LMA, two MAGs, two access points (APs), six Routers and three receivers. The multicast source MN runs VLC media player (VideoLAN client; VideoLAN organization, Paris, France) [53] to provide multicast video multimedia services. The MAG implements MLD proxy function in the BS and runs the PIM-SM protocol in the DMRS. All the other routers in the test-bed support the Routing Information Protocol (RIP), RIP next generation (RIPng) and PIM-SM routing protocols. All the network entities in the test-bed are running Linux operating system (Fedora Core 14) with 2.6.35.6 Linux kernel. Table 3 presents the hardware configuration and IPv6 address configuration.

\subsection{Experimental results}

We mainly test the performance of intra-PMIPv6 domain handover for both the BS and the DMRS under the movement of a multicast source. Using the VLC media player, the mobile multicast source provides multicast data for a certain group. We perform 100 experiments and use the Wireshark (Network Protocol Analyzer; Riverbed Technology, San Francisco, CA, USA) [54] to capture multicast data at the receivers in every experiment. The average value is used to determine the multicast disruption time 



Figure 20 Multicast data flow and throughput of the BS for once handover.

as well as the packet loss on handover for the BS and the DMRS. Since the handover from the SR to the SPT in the BS is so fast that we could not get the results in the SR case accurately, the results of the BS are in the SPT case.

Figure 20 displays the multicast data flow and throughput of the BS for once handover. During the handover of the multicast source $\mathrm{MN}$, the packets received by the multicast receiver will be interrupted until the $\mathrm{MN}$ attached at the new MAG successfully registers with the LMA. As shown in Figure 20, 23 packets were lost during the handover and the total multicast disruption time is $0.287 \mathrm{~s}$, which includes link layer handover delay, PMIPv6 registration delay, and the transmission delay for multicast packets from the source to the receiver. Similarly, Figure 21 shows the multicast data flow and throughput of the DMRS in the case of once handover. As presented in Figure 21, 16 packets were lost during the handover and the total multicast disruption time is $0.312 \mathrm{~s}$, which consists of link layer handover delay,
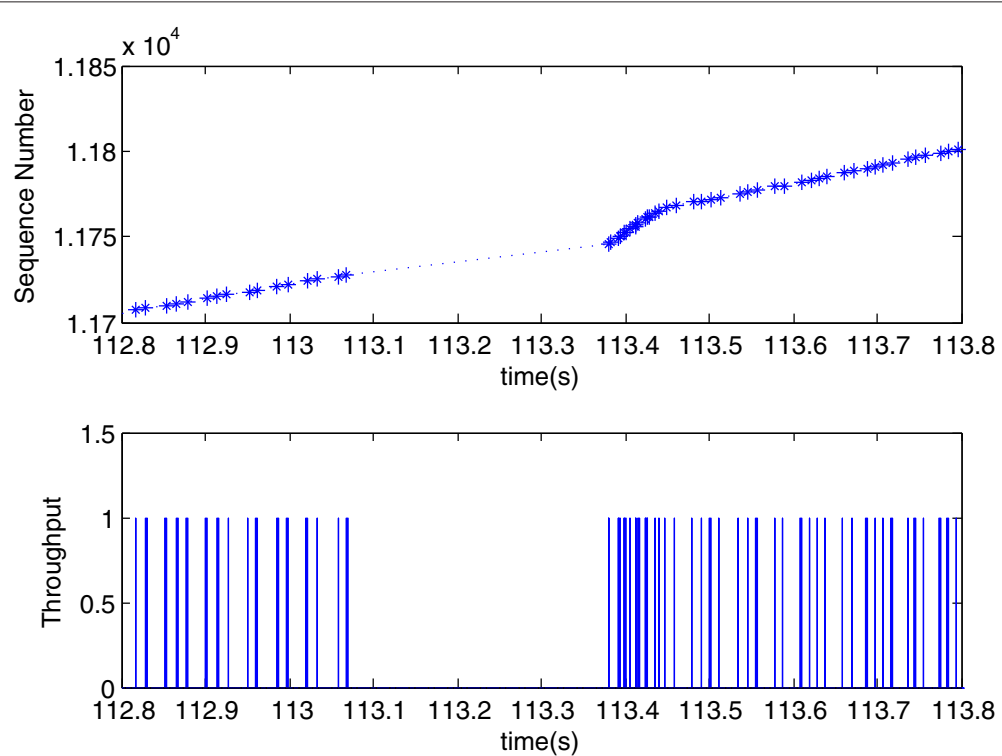

Figure 21 Multicast data flow and throughput of the DMRS for once handover. 


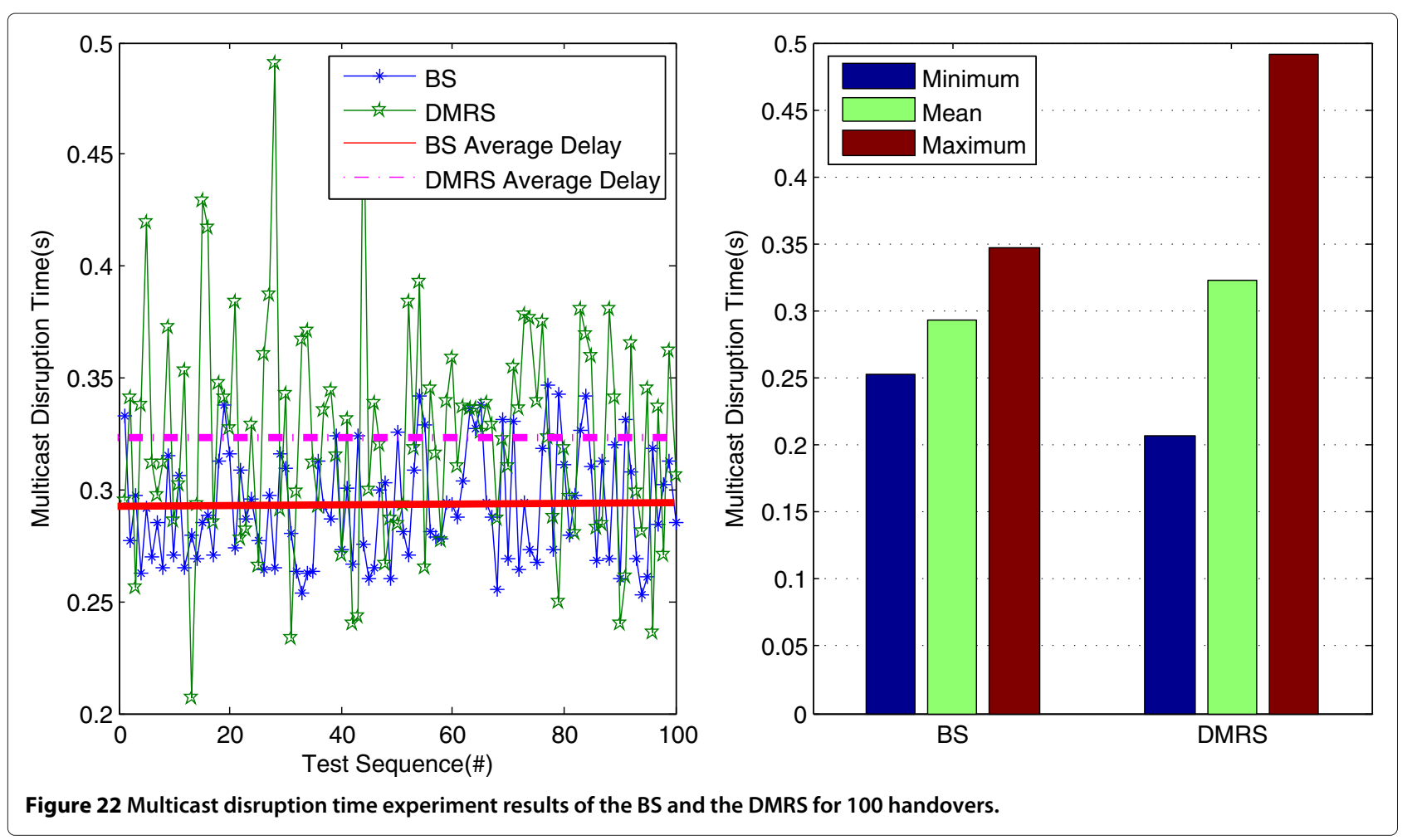

PMIPv6 handover delay, the delay for reestablishing multicast tree from the new MAG to the RP, and the transmission delay for multicast packets from the source to the receiver.

Figures 22 and 23 present the experimental results of the BS and the DMRS for 100 handovers. As shown in Figure 22, the average multicast disruption time in the BS and the DMRS are 0.293 and $0.323 \mathrm{~s}$, respectively. Therefore, the multicast disruption time of the DMRS is $30 \mathrm{~ms}$ longer than the BS in the case of our simple experimental topology. The reason is that when the multicast source MN moves from one MAG to another, the new MAG will act as the DR instead of the previous MAG in the DMRS, but in the BS the LMA always serves as the DR which keeps unchanged, thereby the multicast tree from the DR to the RP needs to be reconstructed for the DMRS but is not needed for the BS. As we can see from Figure 23, there are packet losses during the handover for both the BS and the DMRS. In order to alleviate the packet loss, the option of buffering at the MAGs can be added. In addition, the average packet loss of the DMRS is slightly larger than that of the BS.

\section{Conclusions}

In this paper, the BS and the DMRS are proposed to support the mobile multicast source in PMIPv6 networks, which will lay the foundation for better wireless, mobile, and ubiquitous multimedia applications. The BS is based on the MLD proxies deployed at the MAGs, which is simple to PMIPv6 multicast extensions. However, there exists routing inefficiency problem in the BS, which can be avoided by the DMRS. The reason is that the DMRS can provide a local content distribution service with locally optimized traffic flows. However, the DMRS needs to reestablish the multicast tree whenever the multicast source moves, while the BS does not need to reconstruct the tree, for it has a fixed DR - the LMA. The performance of signaling cost for the proposed schemes is examined by theoretical analysis. Besides, we implement the proposed schemes on the test-bed and conduct the evaluation experiments, which demonstrate the feasibility and validity of the proposed schemes. Based on the signaling cost analysis and the experimental results, we conclude that the total costs in the BS and DMRS are lower than the MIP-BT and MIP-RS, and the DMRS significantly reduces the signaling cost as compared with the BS in the SR case for the optimized routing in the DMRS, while with the path length increment for the DMRS, the BS in the SPT case outperforms the DMRS in terms of signaling cost, multicast disruption time, and packet loss on handover for the SPT handover in the BS. Besides, the signaling cost of all the schemes in the SPT case are lower than those in the SR case. Meanwhile, the analysis results also indicate that a small PMIPv6 domain has the advantage of reducing the total signaling cost for the proposed schemes in the case 


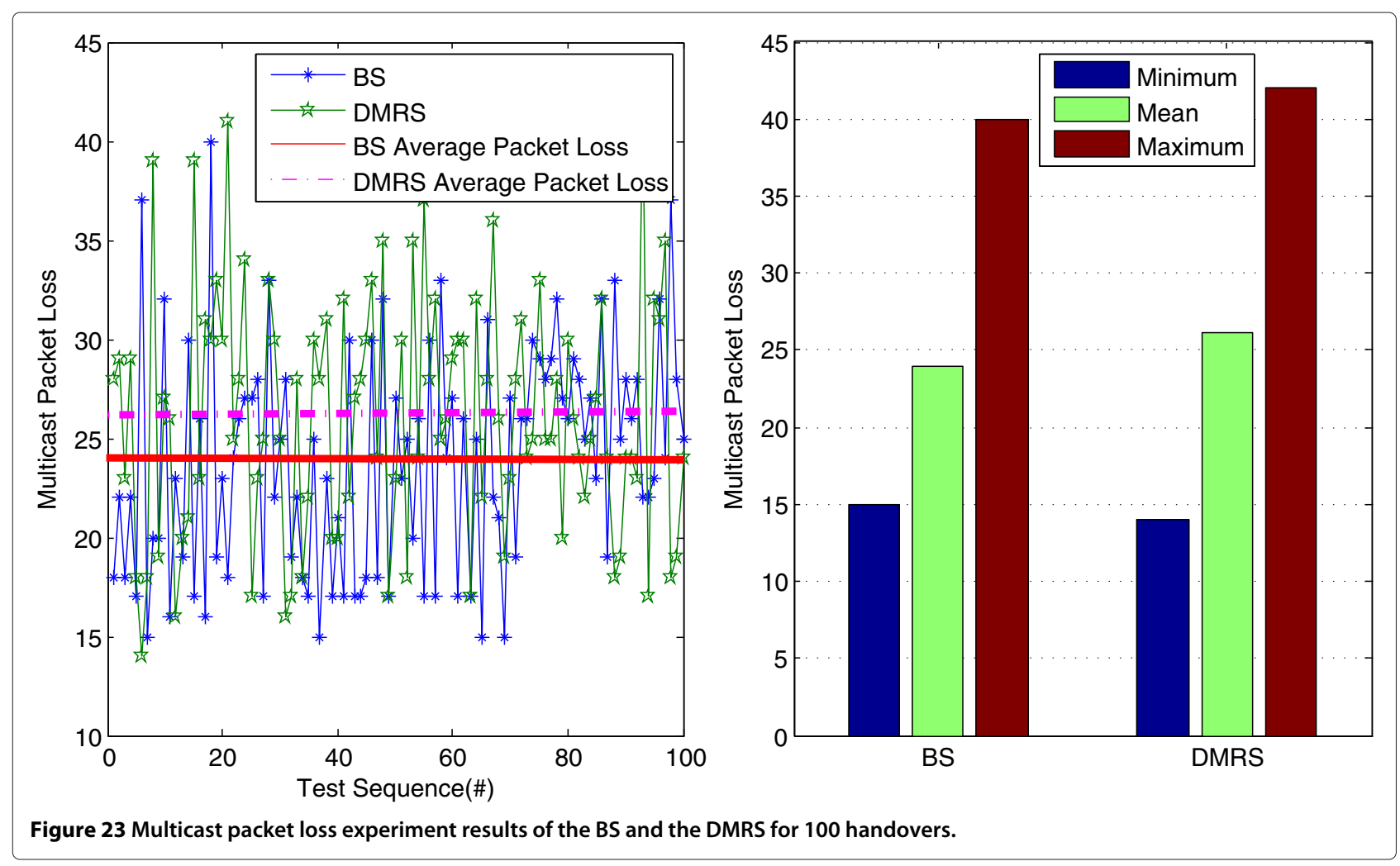

of the static MN, whereas a large PMIPv6 domain has the benefit for reducing the total signaling cost in the case of the dynamic MN.

At the same time, there are still some problems, for example, on how to support the DMRS for multicast source mobility in PMIPv6 networks in the SSM case. In the future, we will study on these problems for better performance and also plan to propose algorithms to select a more optimized solution for mobile multicast source support in PMIPv6 networks from the two proposed schemes based on the different network conditions.

\section{Competing interests}

The authors declare that they have no competing interests.

\section{Acknowledgements}

This work is supported by the National Basic Research Program of China (973 program) under grant no. 2013CB329100, the National Key Technology R\&D Program under grant no. 2012BAH06B01, the Fundamental Research Funds for the Central Universities under grant no. 2013JBM011, the National Natural Science Foundation of China (NSFC) under grant nos. 61100217, 61271202, and 61003283, Beijing Natural Science Foundation under grant no. 4122060, the National High Technology Research and Development Program of China (863 program) under grant no. 2011 AA010701 and the National Key Scientific and Technological Project under grant no. 2013ZX03002001-005.

\section{Author details}

${ }^{1}$ National Engineering Laboratory for NGI Interconnection Devices, School of Electronic and Information Engineering, Beijing Jiaotong University, No. 3 Shang Yuan Cun, Haidian District, Beijing 100044, China. ${ }^{2}$ Department Informatik, HAW Hamburg, Berliner Tor 7, Hamburg 20099, Germany. ${ }^{3}$ State Key Laboratory of Networking and Switching Technology, Beijing University of
Posts and Telecommunications, No. 10 Xi Tu Cheng Road, Haidian District, Beijing 100876, China.

Received: 30 December 2012 Accepted: 15 May 2013

Published: 3 June 2013

\section{References}

1. I Romdhani, M Kellil, HY Lach, A Bouabdallah, H Bettahar, IP mobile multicast: challenges and solutions. IEEE Commun. Surv. Tutorials. 6(1), 18-41 (2004)

2. TC Schmidt, M Waehlisch, G Fairhurst, Multicast mobility in mobile IP version 6 (MIPv6): problem statement and brief survey. IRTF RFC 5757 (MobOpts Research Group, 2010). http://tools.ietf.org/html/rfc5757

3. C Perkins, D Johnson, J Arkko, Mobility Support in IPv6. IETF RFC 6275 (MEXT Working Group, 2011). http://tools.ietf.org/html/rfc6275

4. S Gundavelli, K leung, V Devarapalli, K Chowdhury, B Patil, Proxy mobile IPv6. IETF RFC 5213 (NETLMM Working Group, 2008). http://tools.ietf.org/ $\mathrm{html} / \mathrm{rfc5} 213$

5. K Kong, W Lee, Y Han, M Shin, H You, Mobility management for All-IP mobile networks: mobile IPv6 vs. proxy mobile IPv6. IEEE Wireless Commun. 15(2), 36-45 (2008)

6. TC Schmidt, S Gao, HK Zhang, M Waehlisch, Mobile multicast sender support in proxy mobile IPv6 (PMIPv6) domains, draft-ietf-multimobpmipv6-source-03. IETF Internet Draft (MULTIMOB Working Group, 2013). http://tools.ietf.org/html/draft-ietf-multimob-pmipv6-source-03

7. B Fenner, H He, B Haberman, H Sandick, Internet Group Management Protocol (IGMP)/multicast listener discovery (MLD)-based multicast forwarding ('IGMP/MLD proxying'). IETF RFC 4605 (MAGMA Working Group, 2006). http://www.ietf.org/rfc/rfc4605

8. B Fenner, M Handley, H Holbrook, I Kouvelas, Protocol independent multicast - sparse mode (PIM-SM): protocol specification (revised). IETF RFC 4601 (PIM Working Group, 2006). http://tools.ietf.org/html/rfc4601

9. C Perkins, IP mobility support for IPv4. IETF RFC 3344 (MIP4 Working Group, 2002). http://tools.ietf.org/html/rfc3344 
10. J Lai, W Liao, Mobile multicast with routing optimization for recipient mobility. IEEE Trans. Consum. Electron. 47(1), 199-206 (2001)

11. TG Harrison, WilliamsonC L, WL Mackrell, RB Bunt, Mobile Multicast (MOM) Protocol: multicast support for mobile hosts, in Proceedings of the Third Annual ACM/IEEE International Conference on Mobile Computing and Networking (MOBICOM), (Budapest, pp. 151-160 (26-30 September 1997)

12. CR Lin, KM Wang, Mobile multicast support in IP networks, in Proceedings of the 19th Annual Joint Conference of the IEEE Computer and Communications Societies (INFOCOM), Tel Aviv, pp. 1664-1672 (26-30 March 2000)

13. Y Wang, W Chen, Supporting multicast for mobile hosts. Mobile Netw. Appl. 5(6), 57-66 (2001)

14. YJ Suh, HS Shin, DH Kwon, An efficient multicast routing protocol in wireless mobile networks. ACM Wireless Netw. 7(5), 443-453 (2001)

15. CL Tan, S Pink, MobiCast: a multicast scheme for wireless networks. Netw. Appl. 5(4), 259-271 (2000)

16. CS Jelger, $T$ Noel, Multicast for mobile hosts in IP networks: progress and challenges. IEEE Wireless Commun. 9(5), 58-64 (2002)

17. TC Schmidt, M Waehlisch, Extending SSM to MIPv6-problems, solutions and improvements. Comput. Methods Sci. Technol. 11(2), 147-152 (2005)

18. TC Schmidt, M Waehlisch, Morphing distribution trees-on the evolution of multicast states under mobility and an adaptive routing scheme for mobile SSM sources. Telecommunication Syst. 33(1-3), 131-154 (2006)

19. O Christ, TC Schmidt, M Waehlisch, Towards seamless handovers in SSM source mobility - an evaluation of the Tree Morphing Protocol. Int. J. Internet Protoc. Technol. (IJPT). 3(4), 205-215 (2008)

20. H Lee, S Han, JP Hong, Efficient mechanism for source mobility in source specific multicast, in Proceedings of the 20th International Conference on Information Networking (ICOIN): advances in Data Communications and Wireless Networks, Sendai, pp. 82-91 (16-19 January 2006)

21. H Gossain, S Kamat, DP Agrawal, A framework for handling multicast source movement over mobile IP, in Proceedings of the IEEE International Conference on Communications (ICC) 2002, New York, pp. 3398-3402 (28 April-2 May 2002)

22. B Park, C Lim, An efficient source mobility-based multicast scheme for mobile hosts in mobile-IPv6 networks, in Proceedings of the First IEEE International Symposium on Wireless Pervasive Computing, Phuket, (16-18 January 2006)

23. K Sato, M Katsumoto, T Miki, A New multicast technique supporting source mobility for future vision delivery service, in Proceedings of the 18th IEEE International Conference on Advanced Information Networking and Applications (AINA), Fukuoka, Kyushu, pp. 365-372 (29-31 March 2004)

24. CS Jelger, T Noel, Supporting mobile SSM sources for IPV6, in Proceedings of the IEEE Global Telecommunications Conference (GLOBECOM) 2002, Taipei, (17-21 November 2002)

25. TC Schmidt, M Waehlisch, S Krishnan, Base deployment for multicast listener support in PMIPv6 domains. IETF RFC 6224 (MULTIMOB Working Group, 2011). http://tools.ietf.org/html/rfc6224

26. JF Guan, HC Zhou, CQ Xu, HK Zhang, HB Luo, The performance analysis of the multicast extension support for proxy MIPv6. Wireless Personal Commun. 61(4), 657-677 (2011)

27. S Jeon, N Kang, YH Kim, Mobility management based on proxy mobile IPv6 for multicasting services in home networks. IEEE Trans. Consum. Electron. 55(3), 1227-1232 (2009)

28. Y Li, WT Chen, L Su, DP Jin, LG Zeng, Proxy mobile IPv6 based multicast listener mobility architecture, in Proceedings of the IEEE Wireless Communications and Networking Conference (WCNC) 2009, Budapest, (5-8 April 2009)

29. V Chikarmane, C Williamson, R Bunt, W Mackrell, Multicast support for mobile hosts using mobile IP: design issues and proposed architecture. ACM/Baltzer Mobile Netw. Appl. 3(4), 365-379 (1998)

30. DV Hugo, H Asaeda, B Sarikaya, P Seite, Evaluation of further issues on multicast mobility: potential future work for WG multiMob, draft-von-hugo-multimob-future-work-02. IETF Internet draft (MULTIMOB Working Group, 2010). http://tools.ietf.org/id/ draft-von-hugo-multimob-future-work-02.txt

31. HK Zhang, ZW Yan, S Gao, LL Wang, HW Li, Q Wu, Multicast source mobility support in PMIPv6 network, draft-zhang-multimob-msm-03. IETF
Internet draft (MULTIMOB Working Group, 2011). http://tools.ietf.org/ html/draft-zhang-multimob-msm-03

32. TC Schmidt, M Waehlisch, M Farooq, Mobile multicast sender support in PMIPv6 domains with base multicast deployment, draft-schmidt-multimob-pmipv6-base-source-01. IETF Internet draft (MULTIMOB Working Group, 2011). http://tools.ietf.org/html/draftschmidt-multimob-pmipv6-base-source-01

33. TC Schmidt, S Gao, H Zhang, M Waehlisch. Mobile multicast sender support in PMIPv6 domains, draft-schmidt-multimob-pmipv6-source-00 IETF Internet Draft (MULTIMOB Working Group, 2011). http://tools.ietf. org/html/draft-schmidt-multimob-pmipv6-source-00

34. R Vida, L Costa, Multicast listener discovery version 2 (MLDV2) for IPv6. IETF RFC 3810 (Network Working Group, 2004). http://tools.ietf.org/html/ rfc3810

35. R Wakikawa, S Gundavelli, IPv4 support for proxy mobile IPv6. IETF RFC 5844 (NETLMM Working Group, 2010). http://tools.ietf.org/html/rfc5844

36. M Handley, I Kouvelas, T Speakman, L Vicisano, Bidirectional protocol independent multicast (BIDIR-PIM). IETF RFC 5015 (PIM Working Group, 2007). http://tools.ietf.org/html/rfc5015

37. JC Zuniga, LM Contreras, CJ Bernardos, S Jeon, Y Kim, Multicast mobility routing optimizations for proxy mobile IPV6, draft-ietf-multimob-pmipv6ropt-05. IETF Internet draft (MULTIMOB Working Group, 2013). http:// tools.ietf.org/html/draft-ietf-multimob-pmipv6-ropt-05

38. AS Reaz, PK Chowdhury, M Atiquzzaman, W Ivancic, Signalling cost analysis of SINEMO: seamless end-to-end network mobility, in Proceedings of the First IEEE/ACM International Workshop on Mobility in the Evolving Internet Architecture (MobiArch), In conjunction with IEEE GLOBECOM 2006. San Francisco. (27 November-1 December 2006)

39. Y Fang, Movement-based mobility management and trade off analysis for wireless mobile networks. IEEE Trans. Comput. 52(6), 791-803 (2003)

40. T Brown, S Mohan, Mobility management for personal communications systems. IEEE Trans. Vehicular Technol. 46(2), 269-278 (1997)

41. S Mohan, R Jain, Two user location strategies for personal communications services. IEEE Personal Commun. First Q. 1, 42-50 (1994)

42. S Pack, Y Choi, A study on performance of hierarchical mobile IPv6 in IP-based cellular networks. IEICE Trans. Commun. E87-B(3), 462-469 (2004)

43. X Zhang, JG Castellanos, AT Capbell, P-MIP: Paging extensions for mobile IP. ACM Mobile Netw. Appl. 7(2), 127-141 (2002)

44. J Xie, IF Akyildiz, An optimal location management scheme for minimizing signaling cost in mobile IP, in Proceedings of the IEEE International Conference on Communications (ICC) 2002. New York, pp. 3313-3317 (28 April-2 May 2002)

45. J Xie, IF Akyildiz, A distributed dynamic regional location management scheme for mobile IP, in Proceedings of the 21st Annual Joint Conference of the IEEE Computer and Communications Societies (INFOCOM). New York, pp. 1069-1078 (23-27 June 2002)

46. J Xie, IF Akyildiz, A novel distributed dynamic location management scheme for minimizing signaling costs in mobile IP. IEEE Trans. Mobile Comput. 1(3), 163-176 (2002)

47. HY Tzeng, T Przygienda, On fast address-lookup algorithms. IEEE J. Selected Areas Comm. (JSAC). 17(6), 1067-1082 (1999)

48. B Lampson, V Srinivasan, G Varghese, IP lookups using multiway and multicolumn search. IEEE/ACM Trans. Netw. 7(3), 324-334 (1999)

49. M Woo, Performance analysis of mobile IP regional registration. IEICE Trans. Commun. E86-B(2), 472-478 (2003)

50. J Kempf, Goals for network-based localized mobility management (NETLMM). IETF RFC 4831 (NETLMM Working Group, 2007). http://tools. ietf.org/html/rfc4831

51. Helsinki University of Technology, MIPL - Mobile IPv6 for Linux, MIPL project (Helsinki University of Technology, Helsinki). http://www.mobileipv6.org

52. S Wolke, S Meiling, TC Schmidt, Hamburg University of Applied Sciences, INET research group, mcproxy - multicast proxy for IGMP/MLD (Hamburg University of Applied Sciences, Hamburg, 2012). http://mcproxy.realmv6. org 
53. JB Kempf, VideoLAN Organization, VLC - VideoLAN client (VideoLAN Organization, Paris, 2007). http://www.videolan.org/vlc/

54. G Combs, Riverbed Technology, Wireshark - Network Protocol Analyzer. (San Francisco, CA, USA). http://www.wireshark.org

\section{doi:10.1186/1687-1499-2013-152}

Cite this article as: Wang et al:: Mobile multicast source support in PMIPv6 networks. EURASIP Journal on Wireless Communications and Networking 2013 2013:152.

Submit your manuscript to a SpringerOpen ${ }^{\circ}$ journal and benefit from:

- Convenient online submission

- Rigorous peer review

- Immediate publication on acceptance

- Open access: articles freely available online

- High visibility within the field

- Retaining the copyright to your article

Submit your next manuscript at $\boldsymbol{\wedge}$ springeropen.com 